\title{
Compressive Strength of Tubular Members with Localized Pitting Damage Considering Variation of Corrosion Features
}

\author{
Renhua Wang ${ }^{1 * *}$, Haichao Guo ${ }^{1,}$,, R Ajit Shenoi ${ }^{2}$. \\ wrhchina@163.com \\ 1. Department of Civil Engineering, Jiangsu University of Science and Technology, Zhenjiang 212003, China \\ 2. Southampton Marine and Maritime Institute, University of Southampton, Boldrewood Innovation Campus, \\ Southampton SO16 7QF, UK \\ 3. Taiyuan Urban \& Rural Planning Design Institute, Taiyuan, 030002, China
}

\begin{abstract}
Localized pitting corrosion often occurs on marine and offshore structures in the form of patch corrosion with great uncertainties in the location, size and shape. The variation of corrosion features affects ultimate strength of tubular members significantly, but it is still not well understood. This paper presents a numerical study on tubular members of diverse slenderness ratios to clarify the localized pitting effect on ultimate strength. Numerical analyses were performed based on novel models of pitted members that were calibrated against benchmark column tests. Corrosion pits were randomly introduced on the local outside surfaces of members via stochastic simulation, forming corrosion patches varied in the location, size and shape. Numerical results obtained were regressed to propose a unified empirical formula to predict ultimate strength. It turned out that the shape of the corrosion patch has a significant influence on the ultimate strength. The shape change of the patch can alter failure modes of medium length columns. The reduction of ultimate strength is closely related to the shape ratio of the patch besides the volume loss of corroded material. The unified empirical formula incorporating the shape ratio and the volume loss shows a good ability to predict the experimental results.
\end{abstract}

Keywords: tubular member; localized pitting damage; stochastic simulation; ultimate strength; shape effect. 


\section{Introduction}

Tubular members are one type of structural components of marine and offshore structures in oil, wind, nuclear, hydroelectric, and petrochemical industries [1,2]. It is very common to undergo pitting corrosion for such structures due to long-term exposure to the harsh marine environment [3]. Corrosion products often cover corrosion pits and make them difficult to detect [4]. On the surface, metal loss caused by pitting damage is very small, but it can cause serious degradation in properties of steel material and structural strength, and even sudden structural failure [5]. Therefore, assessing the pitting effect is of importance for maintenance and mitigation on in-service structures. However, it remains a challenge to model random nature of pitting damage, and the uncertainties in corrosion features are rarely concerned $[6,7]$.

Pitting corrosion often produces pits on a member as widely scattered pitting that occurs over the entire surface of a member (widespread pitting), or localized region of pitting (localized pitting) $[8,9]$. Corrosion pits result in an irregular surface on the member. A large number of experimental and numerical studies have been conducted to investigate the effect of widespread pitting on pitted structures including plates [10] and stiffened panels [11,12] under compression, plates under shear [13], and tubular members under compression [1]. To model the uneven surface of a pitted member, corrosion pits were commonly dealt with the circular cavities of the same size and depth, and with a specified regular or irregular distribution. It was shown that widespread pitting causes a significant reduction in ultimate strength, mainly depended on the volume loss of corroded material. In contrast to general (uniform) corrosion, pitting corrosion causes a larger strength reduction under the same level of corrosion damage, despite no consideration in its random nature. In addition, it leads to great variation in the ultimate strength of tubular members, which may follow a normal distribution [1]. Apart from causing the variation and the reduction of ultimate strength, the random pitting damage has a significant influence on the failure modes of pitted structures [14].

The effect of localized pitting on ultimate strength and structural behavior has also been widely concerned. Localized pitting was usually simplified into the form of patch corrosion. The patch had an equivalent section with a locally and uniformly thinned wall thickness in lieu of the irregular one 
of pitting corrosion. Based on the equivalent method, a lot of experiments and numerical studies have been carried out to investigate the ultimate strength and structural behavior of various sections including H-shaped short piles [15], pipelines [16] and tubular members [17]. It was found that the simplified uniform corrosion leads to an overestimation on ultimate strength under the same level of corrosion damage $[18,19]$. This is due to no consideration to the uncertainties of corrosion features (pit size and depth) that can only be described statistically [20,21]. In fact, they can cause great variation in the ultimate strength of pitted structures with widespread pitting [1, 14]. Similarly, the localized pitting causes a reduction of ultimate strength by inducing the volume loss of corroded material. Whereas the localized pitting has extra uncertainties in the location, size and shape of the corrosion patch, as shown in Figure 1. These influential factors significantly influence the ultimate strength and the compressive behavior of tubular members with localized pitting damage, which has been clarified in our previous experimental study [22]. However, their effects were not correlated with the reduction of ultimate strength quantitatively, due to insufficiency that random nature of localized pitting cannot be reflected fully in the limited experimental study. Moreover, to our knowledge, almost no research address this issue systematically.

In this paper, ultimate strength of locally pitted tubular members under axial compression is numerically studied, based on novel models constructed via a modelling method [23]. Localized corrosion pits are randomly introduced on the local surface of the model of each pitted member, forming patch corrosion with various corrosion features varied in the location, size (length and width) and shape. Before performing extensive nonlinear analyses, these models are validated against benchmark column tests. Stochastic simulation is utilized to simulate the random nature of localized pitting. Taking into account the variation of corrosion features, localized pitting effect on compressive behavior and ultimate strength is systematically studied. A unified empirical formula is proposed to predict the ultimate strength of locally pitted tubular members.

\section{Methodology}

Occurrence of the local or overall corrosion should be considered in determining the residual strength of corroded tubular members. However, there is no sufficient information on how to model 
the corrosion damage for strength assessment in the International Standard [24]. Corrosion pits in marine and offshore steel structures are observed to be either cavities or holes being hemispherical or cup-shaped. It was confirmed that the shape of corrosion pits in aged ships is circular, with a ratio of the diameter to depth is in the range of 4:1-10:1 [25], and the maximum pit diameter is in the range of 25-80 $\mathrm{mm}$ [26]. Cylindrical corrosion pits are thus used in this study, as they can yield rational results in experimental and numerical studies of pitted structures $[27,28]$. In order to model localized pitting of tubular members, a modelling method is introduced in this section, which is derived from the method for modelling widespread pitting through a minor adaption [23]. This is followed by a detailed introduction on the variation of corrosion features described by the modified modelling method.

\subsection{Modelling of localized pitting}

Corrosion data derived from normal inspection is generally statistical but not specific, leading to an inaccurate corrosion mapping. The random nature of pitting corrosion can be reflected by random variables relevant to the sizes (radiuses or diameters), depths and distribution locations of corrosion pits. As for corrosion modelling, it is acceptable to determine these variables using the random number method $[3,14]$. The pit size and depth can be decided in terms of actually measured data $[1,14]$. While the distribution location of corrosion pits was thought to comply with a uniform distribution that is typical for the pitting distribution on ship and marine structures [29].

More specifically, the localized pitting was described using two vectors that were named as a shape vector and a location vector. The radiuses, $r$, and depths, $d$, of corrosion pits formed the shape vector $S$, while the pit locations composed the location vector $P$ [14]. Each element of two vectors was determined through performing stochastic simulation. In order to reflect the random nature in pitting distribution, the surface of a tubular member was unfolded into a rectangular plate. The plate had a length of $L$ being the member length, and a width of $\pi D$ being the member perimeter. It was then split into a series of grids with a uniform size. Every corner point of the grids was a possible location to hold a pit, and all the corner points were stored in a vector $U$. To simulate the random pitting distribution, the pits in the vector $S$ were randomly assigned onto some of corner points in 
the vector $U$. The assigned location of each pit was stored in the location vector $P(P \in U)$.

With respect to localized pitting, apart from random nature in the corrosion pits, the corrosion patches with these pits have an extra random nature. It lies in the variation of corrosion features due to the uncertainties in the distribution location (along the length and hoop of a member), the size (length and width) and the shape of a corrosion patch. Therefore, the possible location to hold a pit was limited within the patch. The determination to the locations of pits can be implemented by randomly selecting elements from a limited range of the vector $U$, in line with the size and location of the patch [22], as Eq. (1).

$$
\begin{aligned}
& x_{i}=\operatorname{random}\left(x_{\min }, x_{\max }\right) ; \\
& z_{i}=\operatorname{random}\left(z_{\min }, z_{\max }\right) ;
\end{aligned}
$$

where $x_{\min }$ and $x_{\max }$ denote the start and end columns in the hoop of a member, respectively, while $z_{\min }$ and $z_{\max }$ represent the start and end rows in the length of the member. Figure 2 (a) outlines the flowchart to model localized random pitting. Figure 2 (b) shows a sample of tubular member with localized pitting damage.

Conventionally, the degree of pitting intensity (DOP) and the degree of corroded volume loss (DOV) are used to describe the extent of corrosion damage of the member with widespread pitting $[14,30]$. The two damage indexes are all defined in terms of the entire surface of a member. As for localized pitting, the DOP was supposed to be defined according to locally corroded area (corrosion patch) rather than the entire surface [22], as follows:

$$
D O P=\frac{A_{c}}{A} \times 100(\%)=\frac{1}{\mu_{L} \mu_{P} D L} \sum_{i=1}^{K} r_{i}^{2} \times 100(\%)
$$

Correspondingly, the DOV within the corrosion patch can be defined as [22]:

$$
D O V=\frac{V_{c}}{V} \times 100(\%)=\frac{1}{2 \mu_{L} \mu_{P} R L t} \sum_{i=1}^{K} d_{i} r_{i}^{2} \times 100(\%)
$$

where $A_{c}$ and $V_{c}$ denote the lost area and volume due to corrosion pits within the corrosion patch, respectively, while $A$ and $V$ are the original area and volume without corrosion pits in the corrosion patch. $t$ is the virgin wall thickness of the member, and $R$ is the radius of intermediate surface of the member, equal to $(D-t) / 2 . K$ is the number of the corrosion pits in the corrosion patch, and $r_{i}$ and $d_{i}$ 
are the radius and depth of $i^{\text {th }}$ pit, respectively. $\mu_{L}$ equals to $\left(z_{\min }-z_{\max }\right) / L$ being the ratio of the patch length to member length, and $\mu_{P}$ equals to $\left(x_{\min }-x_{\max }\right) / \pi D$ being the ratio of the patch width to member perimeter. Moreover, an additional parameter, which is named as shape ratio $(S R)$ being the ratio of the length to width of the patch, was used to describe the localized pitting complementally [22], as Eq. (4).

$$
S R=\frac{\mu_{L} L}{\mu_{P} \pi D}=S_{r}\left(\frac{L}{\pi D}\right)
$$

where $S_{r}$ is the relative shape ratio that is normalized as $\mu_{L} / \mu_{P}$ and independent of the dimensions of members. It was used to replace $S R$, so that a unified empirical formula, which is suitable for short columns as well as medium length columns, can be deduced as described in the following sections.

\subsection{Variation of corrosion features}

Pitting corrosion on marine steel structures was influenced by many kinds of factors such as environmental and operational condition, material property and member position $[6,7]$. There are great uncertainties in the distribution pattern, size and shape of corrosion pits [31], but their effcet are rarely examined $[32,33]$. Beyond that, localized pitting has additional uncertainties in its locally pitted area (corrosion patch) that may vary in the distribution, size and shape. Therefore, various pitting patterns in Figure 3 were used to consider these uncertainties.

The nomenclature to identify each pitting pattern consisted of the length, width and location of the corrosion patch, as shown in Figure 3. The patch width was denoted by the angle around the circumference that the corrosion patch subtended. If no specific instruction, the default location of the patch was at mid-height of a member. Otherwise, two capital letters were utilized to indicate the particular patterns that were in the form of a single patch or dual patches that was/were situated at Mid-height, End, Compressive or Tensile side, or Neutral axis.

Figure 3(a) indicates the location change of corrosion patches along the length of a member. The patches contained the same number of corrosion pits that resulted in the same corroded (lost) area and lost volume in these members. In the case, the pitting pattern can be a Single patch with a length of L/4 and a width of $360^{\circ}$ located at Mid-height (L/4-360-SM), or at one End (L/4-360-SE). It might be dual patches situated at both Ends (L/4-360-EE), both Mid-heights (L/4-360-MM), or 
in the form of one at Mid-height and another at End (L/4-360-ME).

Figure 3(b) displays the location change of corrosion patches along the hoop of a member. The patches had the same number of corrosion pits that also leaded to the same level of pitting damage. The interaction of localized pitting and initial deflection was the main interest in such case. A dotted line in Figure 3(b) represents the original cross-section at mid-height of an ideal straight member, while a solid line denotes the final cross-section that was shifted away from the ideal centroid of the section due to initial deflection of the member. Under axial compression, it can induce Tensile behavior on extrados and Compressive behavior on intrados of the member. There were two kinds of pitting patterns such as a single patch and dual patches, both of which had a total length of $L$ and a total width of $90^{\circ}$. The Single patch was situated at the Compressive side (L-90-SC), Tensile side (L-90-ST) or Neutral axes (L-90-SN) of the member. While the dual patches arose in various forms, including one at the Compressive side and another at the Neutral axis (L-90-CN), or one at the Compressive side and another at the Tensile side (L-90-CT).

Figure 3(c) shows the length change of corrosion patches with the same number of corrosion pits. All the patches were located at one end of members, and had the same corroded width of $360^{\circ}$ but with varying lengths. The lengths of the patches were $L / 4$ for $L / 4-360, L / 2$ for $L / 2-360$ and $L$ for L-360, respectively. It is noteworthy that for a corrosion patch with a constant number of corrosion pits, increasing the patch length makes the corroded area increased. Meanwhile, it leads to an ascent in the shape ratio but causes descents in both the DOP and DOV.

Figure 3(d) shows the width change of corrosion patches with a constant number of corrosion pits. It is worth noting that the increase of the patch width also increases the corroded area but with simultaneous descents in the shape ratio, DOP and DOV. All patches had the same corroded length equal to the member length meaning that corrosion pits randomly scatter over the entire length. The width of the patch varied to be $90^{\circ}$ for L- $90,180^{\circ}$ for $\mathrm{L}-180$ and $360^{\circ}$ for L-360, respectively.

The shape change of the corrosion patch involved concurrent changes in the length and width of the patch to ensure that it always had the same corroded area. This differed from the size change in Figure 3(c) and (d) that only involved a separate change in the length or width of the patch. The shape change was thus thought to be a combination of size changes in Figure 3(c) and (d). Hence, 
the pitting patterns for the shape change were not displayed in Figure 3(e), but with only text descriptions. In these cases, a single patch at mid-height of a member varied in the shape, leading to various corrosion scenarios. Two different sizes of corrosion patches were considered, each of which contained the same number of corrosion pits. The smaller one had a corroded area of $\pi D L / 9$, with five pitting patterns, such as Patterns L/9-360, L/6-240, L/3-120, 2L/3-60 and L-40. The larger one had a corroded area of $\pi D L / 4$, also with five pitting patterns, such as Patterns L/4-360, L/3-270, L/2-180, 3L/4-120, and L-90.

\section{Validation of modelling method}

\subsection{Compressive column tests}

Compressive column tests have been carried out on tubular columns to calibrate the proposed modelling method [22]. The test setup and instrument can be found in the previous works [1,22]. Twelve tubular members were tested but only four of them, including one intact and three pitted specimens, were used for the validation of modelling method in this study. Each test specimen had a length of $L=480 \mathrm{~mm}$, a nominal external diameter of $D=60 \mathrm{~mm}$, and a thickness of $t=4.3 \mathrm{~mm}$. Table 1 outlines the information of the four specimens, such as the slenderness ratio $(\lambda)$, the number of corrosion pits $(K)$, the radius $(r)$ and depth $(d)$ of corrosion pits, the DOP and DOV.

Each test specimen was named as "length-width" in terms of the naming scheme previously mentioned in Figure 3, as shown in Table 1. Specimen 0-0 was non-corroded that was the control specimen. Specimen L/4-120 had a corrosion patch of a length of $L / 4$ and a width of $120^{\circ}$. By contrast, the patch in Specimen 3L/4-120 was longer and had a length of 3L/4, while the patch in Specimen L/4-360 was wider and had a width of $360^{\circ}$.

Material properties of the test specimens were determined through prior tensile coupon testing before conducting the compressive column tests. Yield and ultimate strengths were 318 and 485 $\mathrm{MPa}$, respectively, and Young's modulus $(E)$ was $118 \mathrm{GPa}$. The true strain-stress material model from the tensile coupon tests was employed in the validation of numerical modelling.

\subsection{Simulations on column tests}


Numerical modelling on tubular members with localized pitting was performed in the context of APDL of ANSYS software. Numerical models utilized a four-node shell element, Shell 181 that is suitable for analyzing moderately-thick shell structures. The default number of integration points for the single layer model is three, but the number of integration points is changed to a minimum of five during solution when plasticity is present. Fixed-end constraints were applied to two reference nodes at both ends of each member. The reference node located at the centroid of one end section of the member, which was coupled with all the nodes on the end section. For the two reference nodes, all degrees of freedom expect the axial one of the node at loading end were constrained. This was for applying a displacement to mimic axial compression. Material and geometrical nonlinearities were considered in nonlinear analyses by using true stress-strain material model and allowing large deformation to arise. The Newton-Raphson approach was adopted to solve the nonlinear problems.

Three meshing schemes were considered for numerical modelling, including coarse mesh, fine mesh and dense mesh, respectively with 8,16 and 24 elements around one single corrosion pit, as shown in Figure 4. To illustrate their difference clearly, the shell elements of pits were displayed as solids with the shape determined from the real constants via APDL command. Figure 4 shows that the fine mesh scheme yielded better predictions on all test results. Compared with the dense mesh, it needed relatively less consumption of computing resource, but with a smaller relative error between numerical and experimental results. The error was less than $1 \%$ for Specimens $0-0$ and L/4-360, and about 5\% for Specimens L/4-120 and 3L/4-120. Hence, the fine mesh scheme was used throughout the following numerical studies. In addition, Figure 5 and Figure 6 show that numerical models can precisely predicted the load-deformation behavior, failure mode and load-strain behavior for both the intact and pitted test specimens. Particularly, interactive buckling due to the interaction of local buckling and column buckling in pitted specimens was clearly captured using the numerical model, as shown in Figure 6. Overall, the novel models to describe pit detail can be used to reproduce the compressive column tests and can be applied in further numerical studies.

It is believed that the uncertainty in pitting corrosion causes significant variation of ultimate strength $[1,14]$. Therefore, stochastic simualtion was also performed on three pitted test specimens to explore the localized pitting effect on the ultimate strength. One hundred runs of the stochastic 
simualtions were performed on each specimen. Figure 7 shows the simulated ultimate strength of Specimens L/4-120, 3L/4-120 and L/4-360. Obviously, the random nature of pitting distribution caused significant variation of ulitmate strength. The mean values $(\mu)$ and the standard deviations $(\sigma)$ in several interval runs were calculated on the simualtion results, as shown in Table 2 . It is shown that the increase of run number of stochastic simulation on a specimen made the standard deviation slightly reduced. Figure 7 shows that the mean value became stable if the run number was no less than ten. However, more runs almost had no effect on the mean value of ultimate strength but only consumed more computing resource. Therefore, ten runs were thought enough to perform stochastic simulation for each pitted member, which were then employed in the following numerical studies.

\section{Results and discussion}

Based on numerical models validated on tested columns, studies on the effects of corrosion features were extended to a wider range of tubular sections including short columns and medium length columns. Corrosion features shown in Figure 3 were all involved in this section. The short columns had a length of $1200 \mathrm{~mm}$, and the medium length columns had a length of $L=2100 \mathrm{~mm}$. All the columns had the same tubular section of a nominal external diameter, $D=267.4 \mathrm{~mm}$, and of a thickness, $t=9.3 \mathrm{~mm}$, which were tested in [18]. The ratio of the length to the diameter, $L / D$, and slenderness ratio, $\lambda$ were listed in Table 3 , along with pit number, $K$, pit radius, $r$, and pit depth, $d$. It is worth noting that in a specific corrosion patch, the change of pit number $(K=60$ and 120) had the DOP changed, while the change of pit depth $(d=0.25 t, 0.5 t$ and $0.75 t$ mean $25 \%, 50 \%$ and $75 \%$ thickness loss, respectively) had the DOV altered.

Localized pitting damage introduced on the tubular members had the maximum DOP being about $24 \%$ in accordance with the IACS Specification that allows the DOP no more than 25\% [33]. Cylindrical pits with a diameter of $24 \mathrm{~mm}$ were introduced in all the columns. This complied with the inspection data of pits whose maximum diameter is in the range of 25-80 $\mathrm{mm}$ for steel subject to marine immersion corrosion [25]. As for a certain corrosion feature, its effect on ultimate strength and structural behavior was studied under different corrosion scenarios that were derived from 
various combinations of pit depth and pit number shown in Table 3.

All the numerical models in this section were constructed using the above validated modelling method. The same meshing pattern (fine mesh scheme) was also adopted in the numerical analyses. The constraints and the load were exerted by mean of defining the reference nodes as done in the validation of numerical models. Since no specific data of material testing but only the yield strength and tensile strength were provided in [18], bilinear isotropic hardening (BISO) model was adopted for steel material. In terms of the results reported in [18], the steel had a Young's modulus, $E=206$ $\mathrm{GPa}$, a yield stress, $\sigma_{s}=216 \mathrm{MPa}$, an ultimate stress, $\sigma_{u}=373 \mathrm{MPa}$, an ultimate strain, $\varepsilon_{u}=0.2$, and a Poisson's ratio, $v=0.3$. The material and geometric nonlinearities were taken into account in the numerical analyses.

In addition, ten runs of stochastic simulations were performed on each column to account for the random nature of pitting distribution. Regression analysis was then performed on the numerical results of all the columns of diverse slenderness ratios to derive a unified empirical formula.

\subsection{Short columns}

For each pitting pattern shown in Figure 3, two levels of DOPs were considered that were caused by 60 and 120 corrosion pits. At each level of DOP, the pit depth was varied to be $0.25 t$, $0.50 t$ and $0.75 t$, as shown in Table 3. Hence, the effect of a corrosion feature (the location, size or shape of the corrosion patch) on ultimate strength was studied under different corrosion scenarios. The initial ultimate strength of the non-corroded short column was $1812 \mathrm{kN}$. This was a reference strength to define the reduction of ultimate strength in short columns with localized pitting damage. The ratio of the residual strength to the initial strength was denoted by a parameter, $R S_{r}$.

\subsubsection{Location effect}

Location change was reflected by moving the corrosion patch along the length or the hoop of a column, as shown in Figure 3(a) and (b). The corrosion patch was in the form of a single patch or dual patches. For the change along the length, the patch had a total length of $L / 4$ and a total width of $360^{\circ}$ leading to a relative shape ratio, $S_{r}=0.25$, in the columns of L/4-360 series shown in Figure 
3(a). As for the change along the hoop, the patch had a total length of $L$ and width of $90^{\circ}$ yielding a relative shape ratio, $S_{r}=4$, in the columns of L-90 series shown in Figure 3(b). Obviously, the patches in two series of columns had identical corroded area $(\pi D / 4)$ but with different shapes.

Figure 8 shows that localized pitting damage resulted in a remarkable reduction of ultimate strength, and the reduction was highly related to the pit depth and DOP. In other words, the strength reduction was highly depended on DOV, as the DOV was a combination of the pit depth and DOP. The larger DOV, the larger strength reduction. However, disparity in the strength reduction caused by the location change of the patch was tiny, no matter the patch was moved either along the length or along the hoop.

Figure 8(a) shows that the location change along the length made little difference in the reduction of ultimate strength being no more than $2.1 \%$. However, the localized pitting caused great reductions of ultimate strength in most cases. The maximum reduction reached to about $21.6 \%$ for the Pattern L/4-360-EE with 120 pits of pit depth 0.75t. In this case, a single patch was split into dual patches that were situated at both ends of the member. The dual patches leaded to the most serious strength reduction, compared with other pitting patterns, as they were hotbeds for onset of plasticity. In essence, the uneven corroded region at both ends caused an intensive stress interaction between pits. This had the cross-sections at both ends undermined at the same time, weakening the constraint stiffness from fixed-end supports.

The location change along the hoop also had little impact on the reduction of ultimate strength, as shown in Figure 8(b). The maximum difference in the strength reduction caused by the location change was no more than $1.7 \%$. In the worst case, the maximum reduction was about $7.4 \%$ for L90-CN with 120 pits of depth $0.75 t$. It was lower than the maximum reduction in the columns of L/4-360 serious. This resulted from the larger shape ratio of the patches in the columns of L-90 series, since the shape ratio has a significant influence on the ultimate strength.

Overall, the location change of localized pitting damage has a subtle effect on the reduction of ultimate strength under the same DOV, being similar to that with localized uniform corrosion [34].

\subsubsection{Size effect}


Size change was embodied by a separate change in the length or width of the corrosion patch, as shown in Figure 3(c) and (d). Figure 9 displays the variation of ultimate strength due to the size changes. The length change yielded the Max/Min strength reductions of about 19.7\% (for Pattern L/4-360, $S_{r}=0.25$ ) and 8.7\% (for Pattern L-360, $S_{r}=1$ ), making about 10.9\% difference, as shown in Figure 9(a). The width change caused the Max/Min reductions of about 8.7\% (for Pattern L-360, $S_{r}=1$ ) and $5.7 \%$ (for Pattern L-90, $S_{r}=4$ ), with a smaller difference being about 3\%, as shown in Figure 9(b). Therefore, the variation of ultimate strength was slightly more susceptive to the length change of the corrosion patch.

Figure 9 shows that the shape ratio rather than the DOV played a critical role to cause strength variation. In terms of Eqs. (3) and (4), the size change of the corrosion patch leads to simultaneous changes in the DOV and shape ratio. For a corrosion patch with a certain number of corrosion pits, both increases of the length and the width causes a decrease in the DOV. However, the length increase enlarges the shape ratio, while the width increase has it diminished. The reverse effect in the shape ratio has a dominant effect on the variation of ultimate strength. Consequently, as shown in Figure 9, the ultimate strength increased with increase of the length but decreased with increase of the width, despite yielding a reduced DOV. This is due to fact that the shape ratio increased from $S_{r}=0.25$ to $S_{r}=1$ for the length increase, while the shape ratio decreased from $S_{r}=4$ to $S_{r}=1$ for the width increase.

Essentially, the increase of the patch width promotes random corrosion pits to cluster onto a certain cross-section. This stimulates the formation of the weakest cross-section, causing a larger strength reduction. While the increase of the patch length makes the pits disperse along the length, alleviating the confluence of the pits on the section but causing significant variation of ultimate strength. Hence, the ultimate strength of short columns with localized pitting is highly correlated with the size of the corrosion patch. However, the size effect roots from the combined effects of the DOV and the shape ratio, which deserves more investigations.

\subsubsection{Shape effect}

Shape effect was studied upon two types of corrosion patches with different sizes that were 
named as Type S (Small patch) and Type L (Large patch). Type S was smaller and had corroded area of $\pi D L / 9$ with 60 pits, while Type $L$ was larger and had corroded area of $\pi D L / 4$ with 120 pits. Each type of patches consisted of five pitting patterns that had the same corroded area, as listed in Figure 3(e). In Type S, the shape change had the shape ratio varied from $S_{r}=1 / 9$ (Pattern L/9-360) to $S_{r}=9$ (Pattern L-40), while in Type L, the shape change had the shape ratio varied from $S_{r}=1 / 4$ (Pattern L/4-360) to $S_{r}=4$ (Pattern L-90).

Figure 10 shows variation of ultimate strength due to the shape change of the corrosion patch. It is evident that the ultimate strength increased as the shape ratio increased. This is because a longand-narrow patch of a larger shape ratio induced corrosion pits to disperse along the length more separately. This prevented the pits from clustering on the weakest cross-section, causing a smaller strength reduction. In Type S, the Max/Min strength reductions were about 19.3\% for Pattern L/9$360\left(S_{r}=1 / 9\right)$ and about 3.3\% for Pattern L-40 $\left(S_{r}=9\right)$. While in Type L, the Max/Min reductions were about $19.3 \%$ for Pattern L/4-360 $\left(S_{r}=0.25\right)$, and about $7.0 \%$ for Pattern L-90 $\left(S_{r}=4\right)$. This implies that the reduction of ultimate strength was inversely correlated to the shape ratio, and the smaller shape ratio, the larger strength reduction.

Indeed, shape effect on ultimate strength was also reflected in the failure mode, as shown in Figure 10. A corrosion patch in Type L with depth of $0.5 t$ was used to illustrate the failure behavior of short columns with localized pitting. Pattern L/4-360 had a short-and-wide shape of $S_{r}=0.25$, and this patch elicited the column to collapse by local buckling(1)that intensively developed at both edges of the patch. Pattern L-90 had a long-and-narrow shape of $S_{r}=4$, but this patch had no impact to the failure mode. Instead, it induced the collapse by the typical mode in intact short columns that occurred with elephant-foot buckles at both ends(3). Whereas Pattern L/2-180 of a moderate shape ratio of $S_{r}=1$ caused a complex failure mode(2)as an intermediate of the failure modes in Patterns L-90 and L/4-360.

In general, the shape of the corrosion patch has a critical influence on the failure behavior and the ultimate strength of short columns with localized pitting. For the corrosion patch with a shortand-wide shape, the larger patch width can promote more random corrosion pits to cluster onto the cross-section. The confluence of pits intensifies the stress concentration in the locally corroded area, 
stimulating the premature occurrence of plasticity and local buckling.

\subsection{Medium length columns}

Being similar to short columns, each pitting pattern in Figure 3 was studied under two levels of DOPs to investigate the effects of corrosion features on medium length columns. The diverse levels of DOPs were simulated by respectively introducing 105 and 210 pits in the corrosion patch. Under each level of DOP, pit depths were varied in $0.25 t, 0.50 t$ or $0.75 t$, as shown in Table 3 . The ultimate strength of the intact member without corrosion damage was $1687 \mathrm{kN}$, being the reference strength to determine the ratio of residual strength for medium length columns.

\subsubsection{Location effect}

Figure 11 shows the variation of ultimate strength due to location changes of corrosion patches shown in Figure 3(a) and (b). Obviously, the reduction of ultimate strength was highly correlated to the DOV. However, the location change along the length or the hoop of a column made a negligible difference in the strength reduction.

Figure 11(a) shows that in the worst case, the localized pitting caused about $16.8 \%$ reduction of ultimate strength due to the location changes. This took place in Pattern L/4-360-ME with 210 pits of pit depth of $0.75 t$. However, the disparity of strength reduction, caused by the changes from location to location, was tiny, as show in Figure 11. It was only about $1.8 \%$ for the change along the length of the column, and less than $0.9 \%$ for the change along the hoop.

Notably, localized pitting did not affect failure behavior of medium length columns necessarily despite causing serious reduction of ultimate strength, as shown in Figure 12. The initial deflection but not localized pitting damage dominated the failure of the column of $S_{r}=4$. It always collapsed by column buckling, no matter the corrosion patch was situated at the intrados (Pattern L-90-SC) or the extrados (Pattern L-90-ST) of the column.

\subsubsection{Size effect}

Figure 13 illustrates the variation of ultimate strength due to size changes of corrosion patches shown in Figure 3(c) and (d). Obviously, it was also closely related to the pit depth, DOP and DOV. 
Moreover, the length change caused more pronounced variation of strength reduction than the width change. As for the length change, the maximum strength reduction was about $16.0 \%$ for Pattern L/4-360, while the minimum one was 9.7\% for Pattern L-360, as shown in Figure 13(a). The length change of the patch resulted in great variation of ultimate strength, but it diminished as pit depth decreased. In the case that the pit depth decreased to be $0.25 t$, the strength reduction was only $0.9 \%$ for Pattern L/4-360, and 1.9\% for Pattern L-360, with a small difference.

As noted previously, there is a reverse effect on the shape ratio due to the changes of the patch length and width. That is, the length increase causes a larger shape ratio but the width increase yields a smaller shape ratio. Such change in the shape ratio played an important role to affect the strength reduction, especially at the higher level of DOV, as shown in Figure 13. However, the effect weakened with the decrease of the DOV.

Similarly, the corrosion patch of a larger width tends to cause pit cluster on a certain section. This initiates early local buckle on member wall, leading to a larger strength reduction. Notably, the size effect results from the combination of the shape ratio and the volume loss of corroded material, which deserves more studies.

\subsubsection{Shape effect}

Figure 14 shows variation of ultimate strength due to the shape change of the corrosion patch. Evidently, the ultimate strength increases with the increase of the shape ratio. The greatest variation of strength reduction occurred in the case that the corrosion patch contained 210 pits of pit depth of 0.75t. The Max/Min strength reductions were about $16.2 \%$ for Pattern L/4-360 $\left(S_{r}=0.25\right)$ and 7.7\% for Pattern L-90 $\left(S_{r}=4\right)$, respectively. However, the disparity of strength reduction diminished with the decrease of the DOV.

Moreover, the variation of ultimate strength displayed a slight fluctuation, as shown in Figure 14. This was ascribed to the transition of failure mode caused by the shape change of the corrosion patch, as shown in Figure 15. It is shown that the failure mode was influenced not only by the shape ratio but also by the level of corrosion damage. Seen from Figure 15, the short-and-wide patch of $S_{r}$ $=0.25$ (Pattern L/4-360) caused local buckling under various pit depths $(0.25 t, 0.75 t)$. Whereas the 
long-and-narrow patch of $S_{r}=4$ (Pattern L-90) always induced column buckling, due to the leading role of initial deflection as described previously on Figure 12. However, the patch of a moderate shape ratio of $S_{r}=1.0$ (Pattern L/2-180) had a varying effect on the failure mode highly depending on the pit depth. For the smaller pit depth $(0.25 t)$, the column failed by column buckling, while interactive buckling occurred for the larger pit depth $(0.75 t)$. There was a critical shape ratio, $S_{r}=$ 1.0 that can ignite the transition of failure mode with the change of pitting damage. The critical value corresponds to the mutant sites of the fluctuated curves shown in Figure 14.

Overall, the shape change of corrosion patches plays an important role to influence the failure behavior and the compressive strength of medium length columns. This is because the shape change of the corrosion patch affects the distribution of corrosion pits. For a short-and-wide patch, the pits tend to cluster on the same section of the damaged column. This impairs the effective cross-section and induces early local buckling due to the stress interaction within pit colony.

\subsection{Empirical formula}

The DOV has been widely accepted as a dominant parameter to influence ultimate strength of pitted members. Numerical studies in previous sections show that the shape ratio, $S_{r}$, is also a key parameter that needs to be involved in ultimate strength assessment. Therefore, this section attempts to incorporate the shape ratio and the DOV to develop a unified empirical formula for prediction of ultimate strength of locally pitted members.

All numerical results of ultimate strength relevant to the changes of location, size and shape of corrosion patches were plotted against the DOV, in order to display insufficiency if only considering the DOV in determining the ultimate strength of locally pitted members. The effects of the size and location are shown in Figure 16 and Figure 17, respectively, for short columns and medium length columns. The shape effect on two types of columns are plotted in Figure 18. Obviously, significant variation of ultimate strength arose for a certain corrosion feature, resulting from the random nature of pitting damage. In particular, the shape effect encounters greater variation of ultimate strength under the higher level of volume loss, as it has a wider range of scattered data. In addition, Figure 17 and Figure 18 consistently show that the ultimate strength is highly associated with not only the 
DOV but also the shape ratio. However, the size effect (length and width of the corrosion patch) results from the combination of the DOV and shape ratio that are difficult to separate. Therefore, only these results from the shape effect in Figure 18 were used to develop the empirical formula to account for the shape effect.

The shape effect has been demonstrated in Figure 10 and Figure 14, respectively, for short columns and medium length columns, using pitting patterns as the $x$-axis. Data in the two figures were transformed, using the shape ratio to replace the pitting pattern as the $x$-axis, as shown in Figure 19 and Figure 20. As for short columns, its failure is governed by local buckling. However, medium length columns have complex failure behavior likely being column buckling, interactive buckling or even local buckling. For a medium length column, local buckling can play a dominant role only if the shape ratio is smaller than 1.0. In this case, it can be evaluated as a short column, and its result was considered to have been included in the results of short columns in Figure 19. Therefore, the data of medium length columns derived from Figure 14 were truncated at the shape ratio smaller than 1.0, as shown in Figure 20. Regression analyses were then performed on Figure 19 and Figure 20, yielding a logarithmic expression as Eq. (5).

$$
R S_{r}=\alpha \ln \left(S_{r}\right)+\beta
$$

where $R S_{r}$ is the ratio of ultimate strength of the pitted member in contrast to the intact one; $\alpha$ and $\beta$ are the coefficients of logarithmic curves depending on the fitting curves in Figure 19 and Figure 20. The two coefficients were determined to be power expressions plotted in Figure 21, as Eq. (6).

$$
\begin{aligned}
& \alpha=0.0068 D O V^{9.6788} \\
& \beta=0.9955 D O V^{-0.659}
\end{aligned}
$$

The normalized ultimate strength of locally pitted members in Eq. (5) can be determined by:

$$
R S_{r}=0.0068 D O V^{9.6788} \ln \left(S_{r}\right)+0.9955 D O V^{-0.659}
$$

As for a given DOV, Eq. (7) can be used to predict the ultimate strength of the compressive columns with localized pitting as a function of shape ratio of the corrosion patch. A comparison of analytical results from Eq. (7) and experimental results is shown in the fifth Column and the fourth Column of Table 4. In contrast to the column tests in this paper, the relative errors are less than $3.9 \%$; while for the short column tests in the existing studies [18], the relative errors are less than $5 \%$ for most cases, 
as shown in the sixth Column of Table 4. The unified empirical formula can predict the test results of tubular members with localized pitting damage precisely.

\section{Conclusion}

This paper presents a comprehensive numerical study on the effects of corrosion features on the ultimate strength and failure behavior of locally pitted tubular members under compression. It was carried out based on novel models to depict pit details that were validated against the devised compressive column tests. Corrosion features included the location, size and shape of the corrosion patch. These features have different impacts to the ultimate strength. In the worst case, localized pitting can cause a decrease of the ultimate strength being more than $20 \%$. The shape change of the corrosion patch affects the strength reduction significantly, leading to great strength variation being about $16 \%$ for short columns and about $10 \%$ for medium length columns. It may cause a transition of failure mode in medium length columns. The shape ratio of the patch is a key factor that needs to be considered, for ultimate strength assessment, together with the volume loss of corroded material. The unified empirical formula incorporating the two factors shows a good ability to predict the test results.

\section{Acknowledgement}

The first author would like to thank the National Natural Science Foundation of China [grant number 51879124] for the financial support, and appreciate Mr. Chaoming Shen from the Engineering Mechanics Laboratory at the Jiangsu University of Science and Technology for his assistance during the experimental study.

\section{References}

[1] Wang R, Shenoi RA. Experimental and numerical study on ultimate strength of steel tubular members with pitting corrosion damage. Marine Structures. 2019;64:124-37.

[2] Ahmadi H, Zavvar E. Degree of bending (DoB) in offshore tubular KT-joints under the axial, in-plane bending (IPB), and out-of-plane bending (OPB) loads. Applied Ocean Research. 2020;95:102015.

[3] Silva JE, Garbatov Y, Guedes Soares C. Ultimate strength assessment of rectangular steel plates subjected to a random localised corrosion degradation. Engineering Structures. 2013;52:295-305.

[4] Chouchaoui BA, Pick RJ. Behaviour of longitudinally aligned corrosion pits. International Journal of Pressure Vessels and Piping. 1996;67:17-35.

[5] Cai J, Jiang X, Lodewijks G. Residual ultimate strength of offshore metallic pipelines with structural damage - a literature review. Ships and Offshore Structures. 2017;12:1037-55. 
[6] Bhandari J, Khan F, Abbassi R, Garaniya V, Ojeda R. Modelling of pitting corrosion in marine and offshore steel structures - A technical review. Journal of Loss Prevention in the Process Industries. 2015;37:39-62.

[7] Wang Y, Wharton JA, Shenoi RA. Ultimate strength analysis of aged steel-plated structures exposed to marine corrosion damage: A review. Corrosion Science. 2014;86:42-60.

[8] ABS. Buckling and ultimate strength assessment for offshore structures. Houston: American Bureau of Shipping; 2014.

[9] ASME-B31G. Manual for determining the remaining strength of corroded pipelines. Washington: American Society of Mechanical Engineers; 2012.

[10] Jiang X, Guedes Soares C. Ultimate capacity of rectangular plates with partial depth pits under uniaxial loads. Marine Structures. 2012;26:27-41.

[11] Sultana S, Wang Y, Sobey AJ, Wharton JA, Shenoi RA. Influence of corrosion on the ultimate compressive strength of steel plates and stiffened panels. Thin-Walled Structures. 2015;96:95-104.

[12] Zhang J, Shi XH, Guedes Soares C. Experimental analysis of residual ultimate strength of stiffened panels with pitting corrosion under compression. Engineering Structures. 2017;152:70-86.

[13] Zhao Z, Zhang N, Wu J, Gao Y, Sun Q. Shear capacity of steel plates with random local corrosion. Construction and Building Materials. 2020;239:117816.

[14] Wang R, Shenoi RA, Sobey A. Ultimate strength assessment of plated steel structures with random pitting corrosion damage. Journal of Constructional Steel Research. 2018;143:331-42.

[15] Karagah H, Shi C, Dawood M, Belarbi A. Experimental investigation of short steel columns with localized corrosion. Thin-Walled Structures. 2015;87:191-9.

[16] Mohd MH, Lee BJ, Cui Y, Paik JK. Residual strength of corroded subsea pipelines subject to combined internal pressure and bending moment. Ships and Offshore Structures. 2015;10:554-64.

[17] Nazaria M, Khedmati MR, Khalaj AF. A numerical investigation into ultimate strength and buckling behavior of locally corroded steel tubular members. Latin American Journal of Solids and Structures. 2014;11:1063-76.

[18] Ahn J-H, Choi WR, Jeon SH, Kim S-H, Kim I-T. Residual compressive strength of inclined steel tubular members with local corrosion. Applied Ocean Research. 2016;59:498-509.

[19] Wang H, Yu Y, Yu J, Xu W, Chen H, Wang Z, et al. Effect of pitting defects on the buckling strength of thick-wall cylinder under axial compression. Construction and Building Materials. 2019;224:226-41.

[20] Melchers RE. Statistical characterization of pitting corrosion - Part 1: Data analysis. Corrosion. 2005;61:655-64

[21] Melchers RE. Statistical characterization of pitting corrosion - Part 2: Probabilistic modeling for maximum pit depth. Corrosion. 2005;61:766-77.

[22] Wang R, Guo H, Shenoi RA. Experimental and numerical study of localized pitting effect on compressive behavior of tubular members. Marine Structures. 2020;72:102784.

[23] Wang R, Sun J, Fang Y. The parameterization construction method of numerical model of cylindrical shells with random pitting corrosion. China. China Patent Bureau: 2018, ZL201510238911.2.

[24] ISO19902. Petroleum and natural gas industries - Fixed steel offshore structures. Geneva: the International Organization for Standardization; 2007.

[25] Nakai T, Matsushita H, Yamamoto N, Arai H. Effect of pitting corrosion on local strength of hold frames of bulk carriers (1st report). Marine Structures. 2004;17:403-32.

[26] Paik JK, Lee JM, Ko MJ. Ultimate compressive strength of plate elements with pit corrosion wastage. Journal of Engineering for the Maritime Environment. 2003;217:185-200.

[27] Shi XH, Zhang J, Guedes Soares C. Numerical assessment of experiments on the ultimate strength of stiffened panels with pitting corrosion under compression. Thin-Walled Structures. 2018;133:52-70.

[28] Feng L, He J, Hu L, Shi H, Yu C, Wang S, et al. A parametric study on effects of pitting corrosion on steel plate's ultimate strength. Applied Ocean Research. 2020;95:102026.

[29] Paik JK, Lee JM, Ko MJ. Ultimate shear strength of plate elements with pit corrosion wastage. Thin-walled Structures. 2004;42:1161-76.

[30] Zhang Y, Huang Y, Zhang Q, Liu G. Ultimate strength of hull structural plate with pitting corrosion damnification under combined loading. Ocean Engineering. 2016;116:273-85.

[31] Yamamoto N, Ikegami K. A study on the degradation of coating and corrosion of ship's hull based on the probabilistic approach. Journal of Offshore Mechanics and Arctic Engineering. 1998;120:121-8.

[32] Zhang Y, Huang Y, Wei Y. Ultimate strength experiment of hull structural plate with pitting corrosion damage under unaxial compression. Ocean Engineering. 2017;130:103-14.

[33] IACS. Requirements concerning survey and certification. London: International Association of Classification Societies; 2016.

[34] Shi C, Karagah H, Dawood M, Belarbi A. Numerical investigation of H-shaped short steel piles with localized 
severe corrosion. Engineering Structures. 2014;73:114-24.

$-21-$ 


\section{List of Figures and Tables}

Figure 1 Locally pitted steel columns under marine environment [22]. (a) Tubular section; (b) H section.

Figure 2 Simulated tubular members with localized pitting. (a) Flowchart of numerical modelling; (b) sample model.

Figure 3 Variation of corrosion features of locally pitted members. (a) Location change along the length; (b) location change along the hoop; (c) length change; (d) width change; (e) shape change. Figure 4 Ultimate strength of test specimens derived from different meshing patterns.

Figure 5 Comparison between experimental and numerical results of the intact member.

Figure 6 Comparison between experimental and numerical results of the pitted member.

Figure 7 Convergence of stochastic simulation on the tested pitted members.

Figure 8 Variation of ultimate strength due to location change of corrosion patches in short columns.

(a) Change along the length; (b) change along the hoop.

Figure 9 Variation of ultimate strength due to size change of corrosion patches in short columns. (a) Length change; (b) width change.

Figure 10 Variation of ultimate strength due to shape change of corrosion patches in short columns. Figure 11 Variation of ultimate strength due to location change of corrosion patches in medium length columns. (a) Change along the length; (b) change along the hoop.

Figure 12 Failure mode dominated by initial deflection of a medium length column.

Figure 13 Variation of ultimate strength due to size change of corrosion patches in medium length columns. (a) Length change; (b) width change.

Figure 14 Variation of ultimate strength due to shape change of corrosion patches in medium length columns.

Figure 15 Failure mode transition due to shape change of corrosion patches in a medium length column.

Figure 16 Variation of ultimate strength due to changes of location and size of corrosion patches in short columns.

Figure 17 Variation of ultimate strength due to changes of location and size of corrosion patches in medium length columns.

Figure 18 Variation of ultimate strength due to shape change of corrosion patches.

Figure 19 Relation of ultimate strength of short columns with shape ratios of corrosion patches. (a) $\pi D L / 9$ (60 pits); (b) $\pi D L / 4$ (120 pits). 
Figure 20 Relation of ultimate strength of medium length columns with shape ratios of corrosion patches. (a) $\pi D L / 9$ (105 pits); (b) $\pi D L / 4$ (210 pits).

Figure 21 Coefficients of empirical formula of ultimate strength.

Table 1 Test specimens with/without corrosion pits.

Table 2 Results of stochastic simulation on ultimate strength $(\mathrm{kN})$ of test specimens.

Table 3 Model parameters of tubular sections in numerical analyses.

Table 4 Comparison between predicted and experimental results. 


\section{Figures}

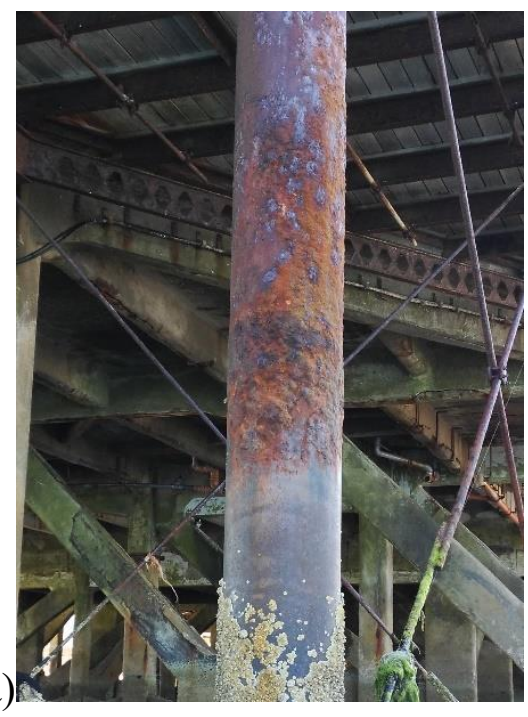

(b)

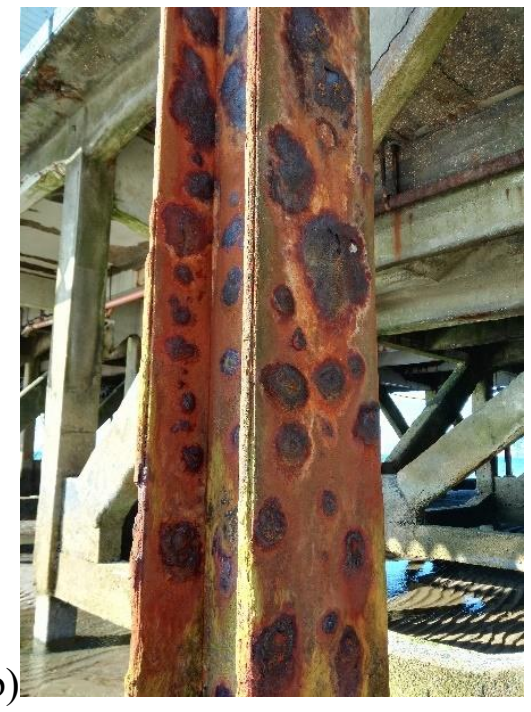

Figure 1 Locally pitted steel columns under marine environment [22]. (a) Tubular section; (b) H section.
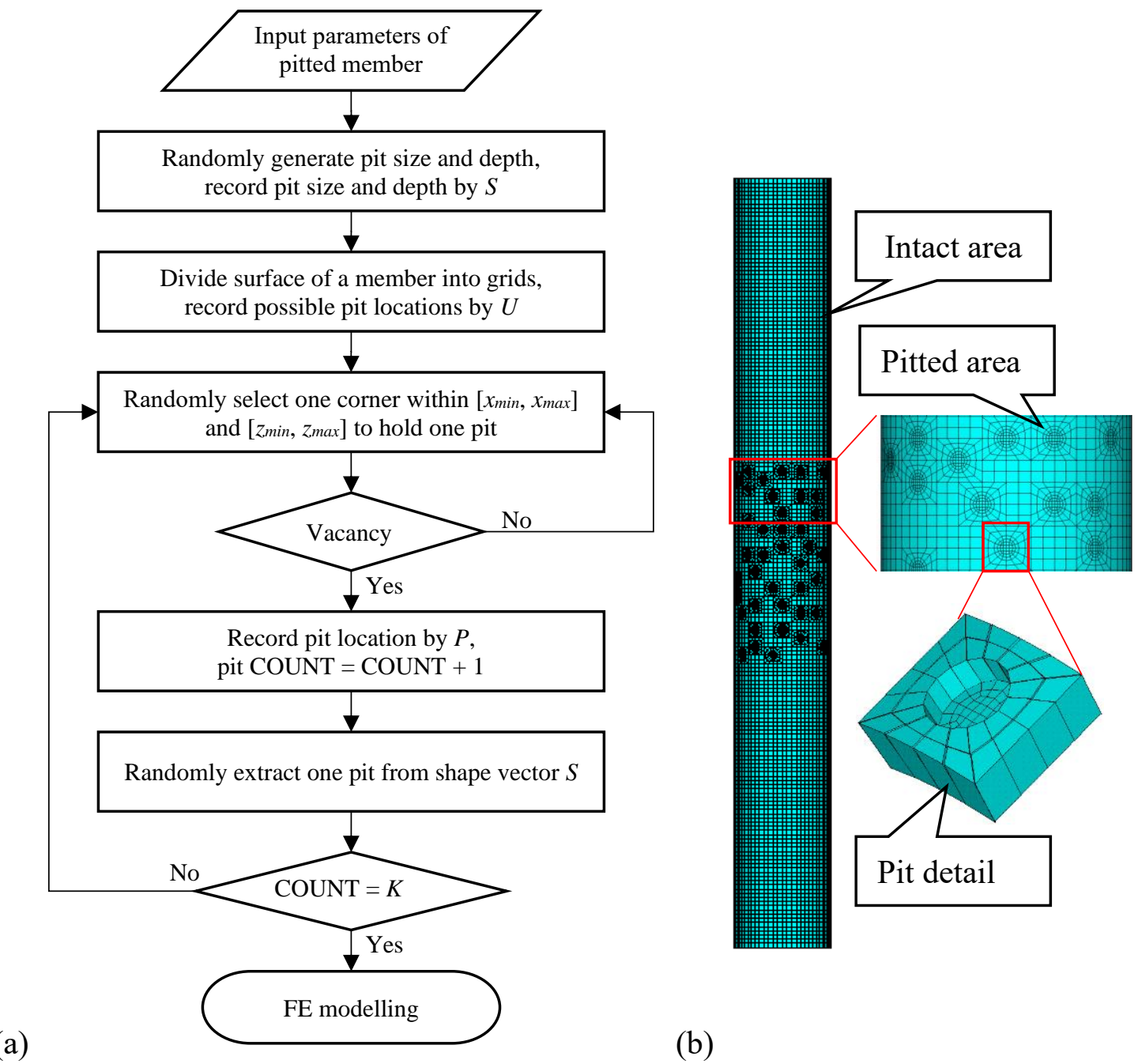

(b)

Figure 2 Simulated tubular members with localized pitting. (a) Flowchart of numerical modelling; 
(b) sample model.
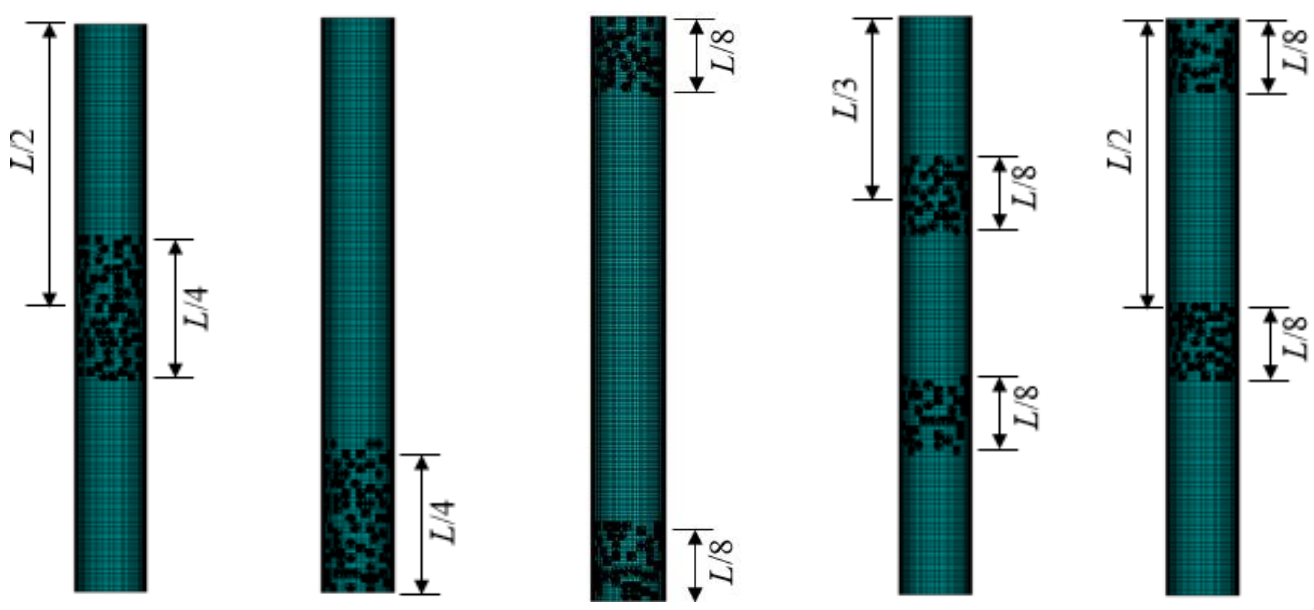

(a)
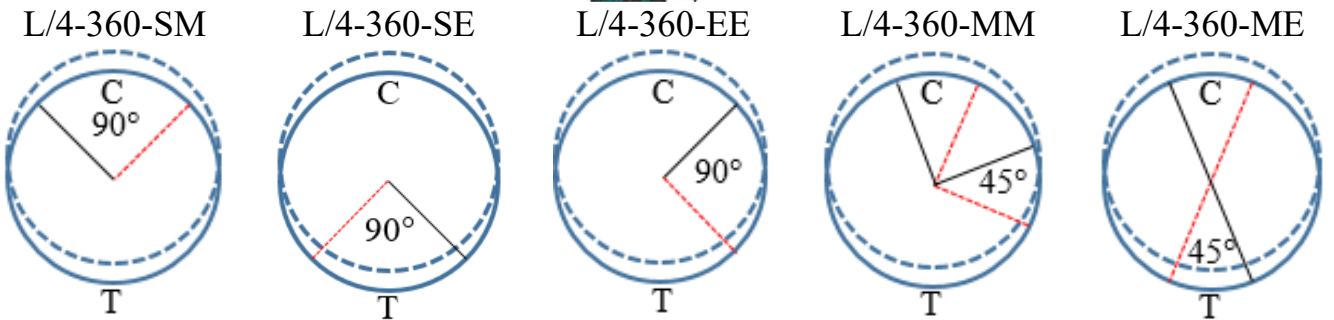

(b)

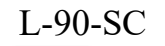

L-90-ST

L-90-SN
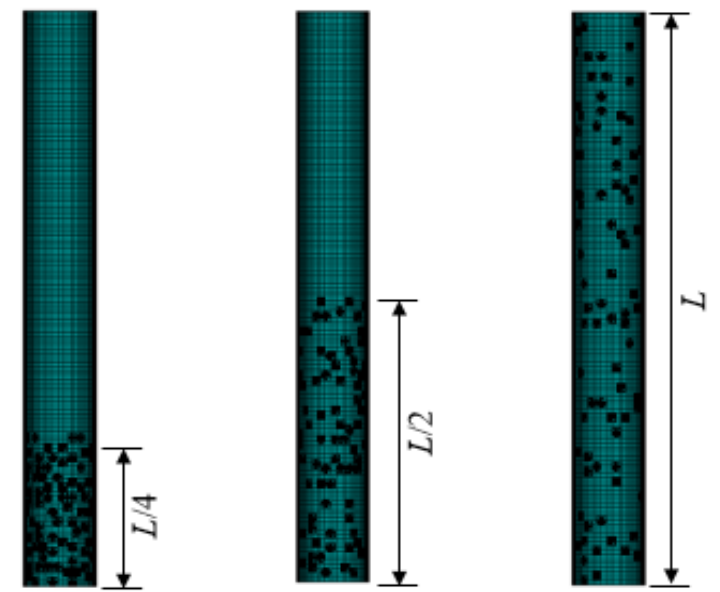

(c)
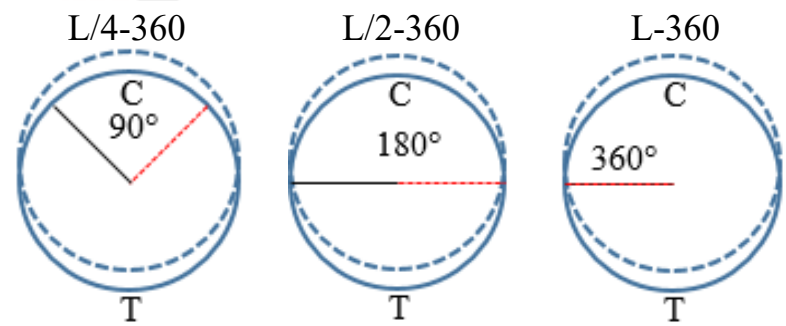

(d)

$$
\text { L-90 }
$$

L-180

L-360

$\mathrm{L} / 9-360$

L/6-240

L/3-120

2L/3-60

L-40

(e) $\quad$ L/4-360

L/3-270

L/2-180

3L/4-120

L-90

Figure 3 Variation of corrosion features of locally pitted members. (a) Location change along the length; (b) location change along the hoop; (c) length change; (d) width change; (e) shape change. 


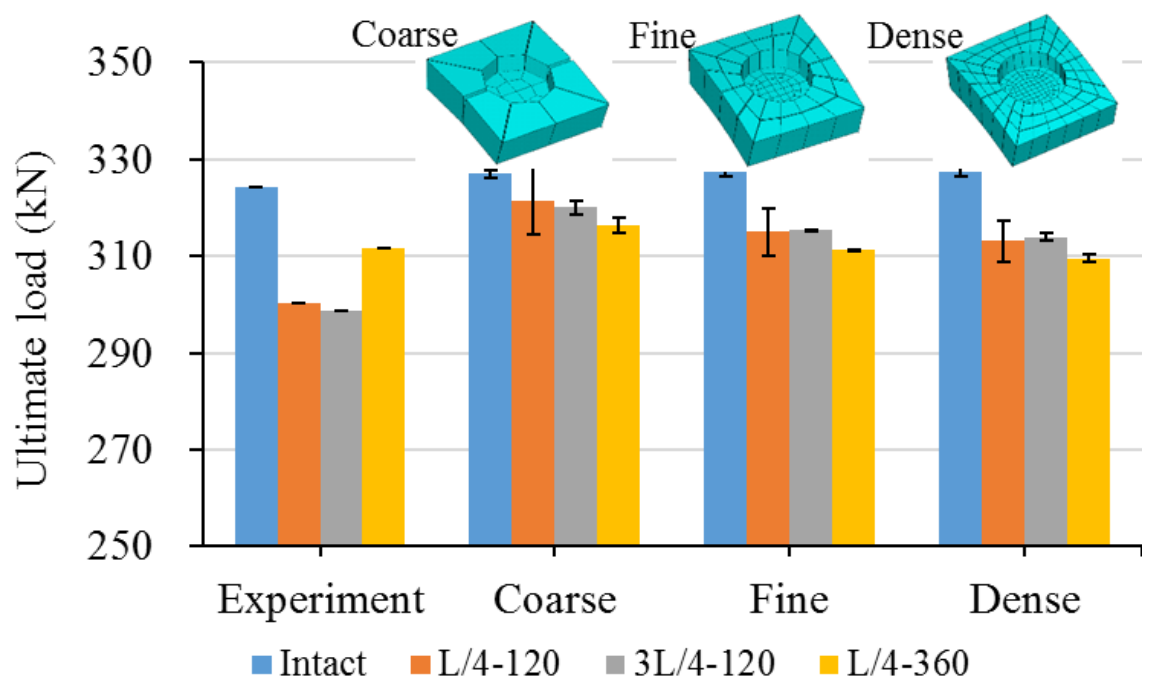

Figure 4 Ultimate strength of test specimens derived from different meshing patterns.
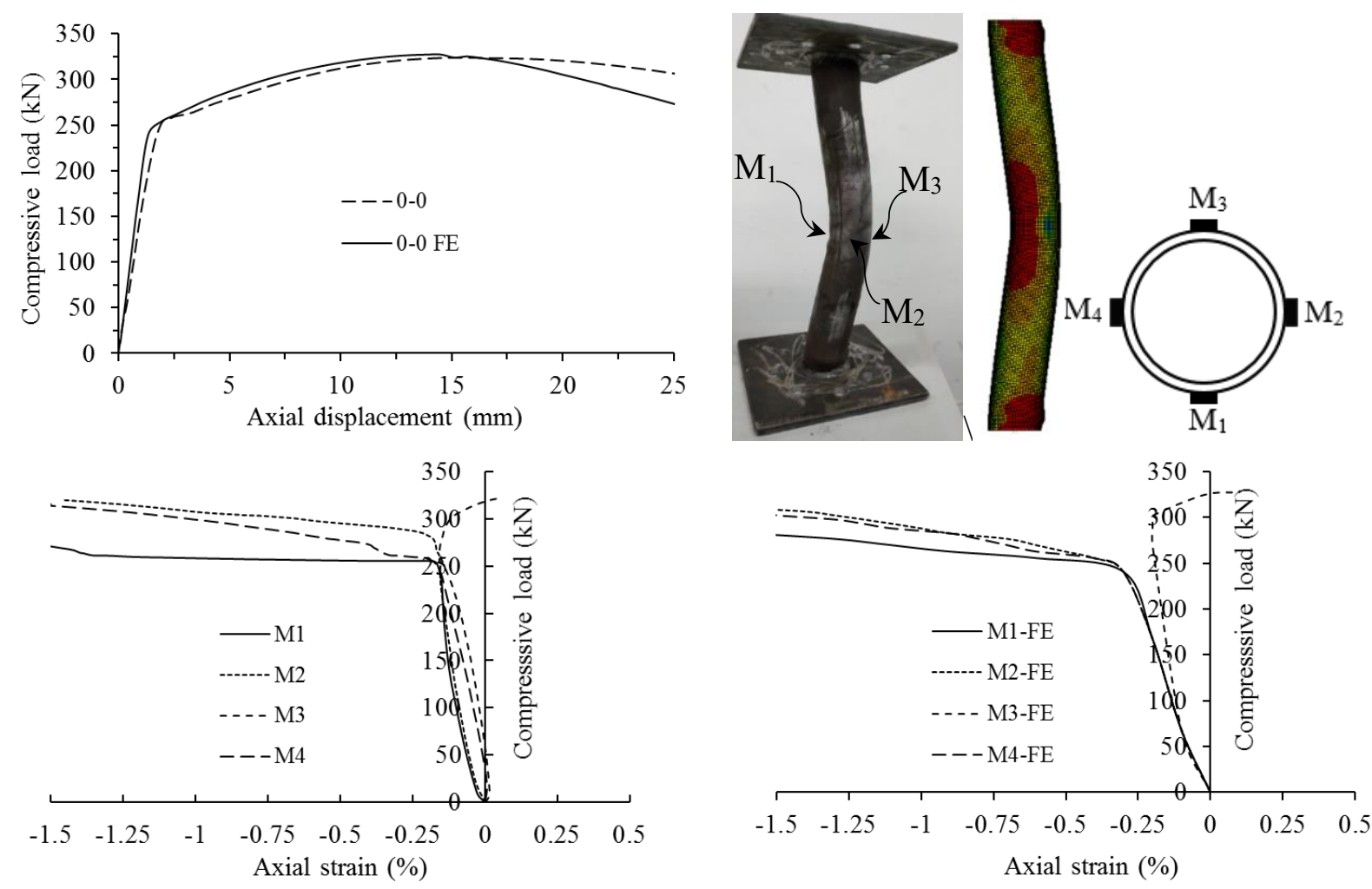

Figure 5 Comparison between experimental and numerical results of the intact member. 

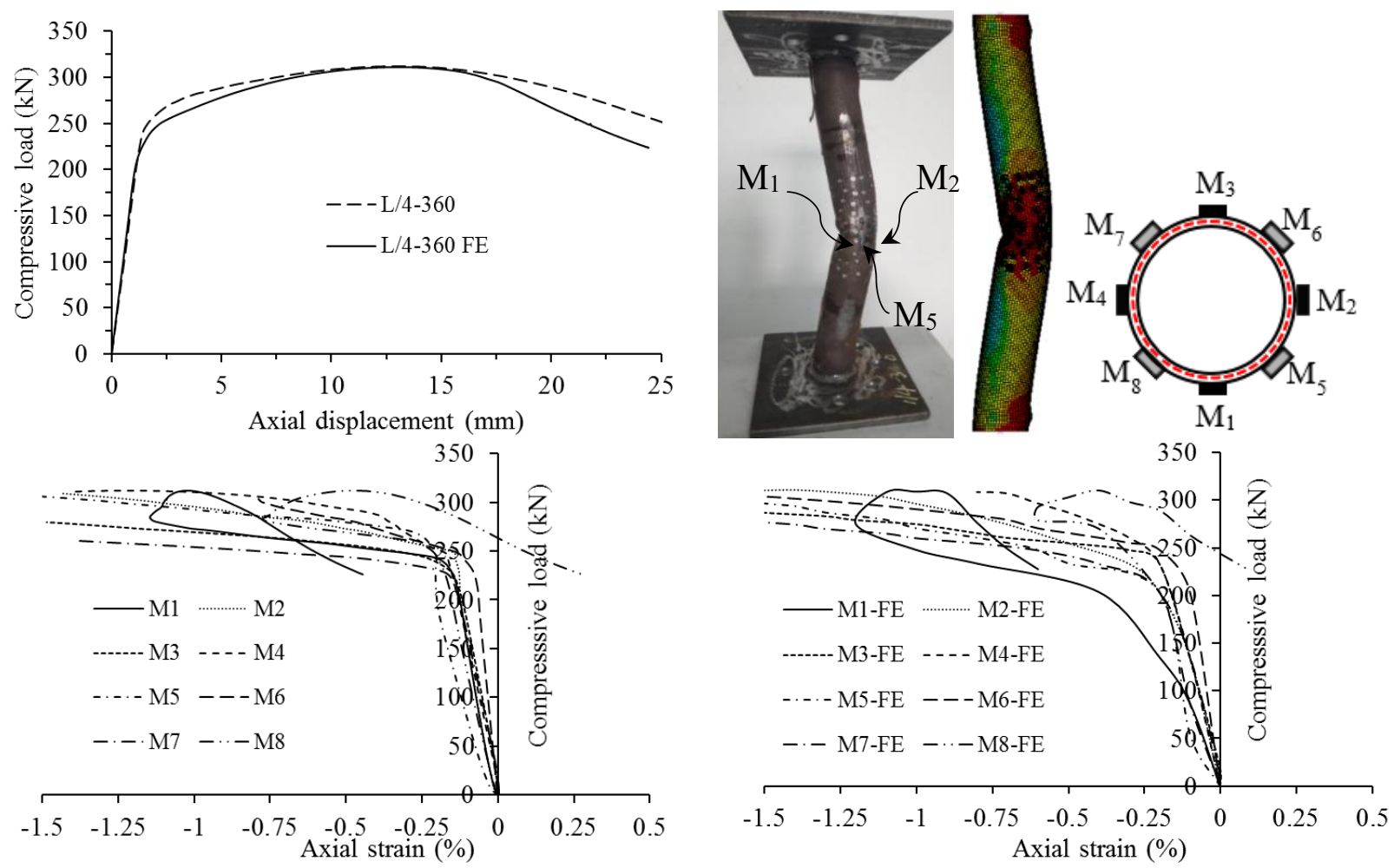

Figure 6 Comparison between experimental and numerical results of the pitted member.

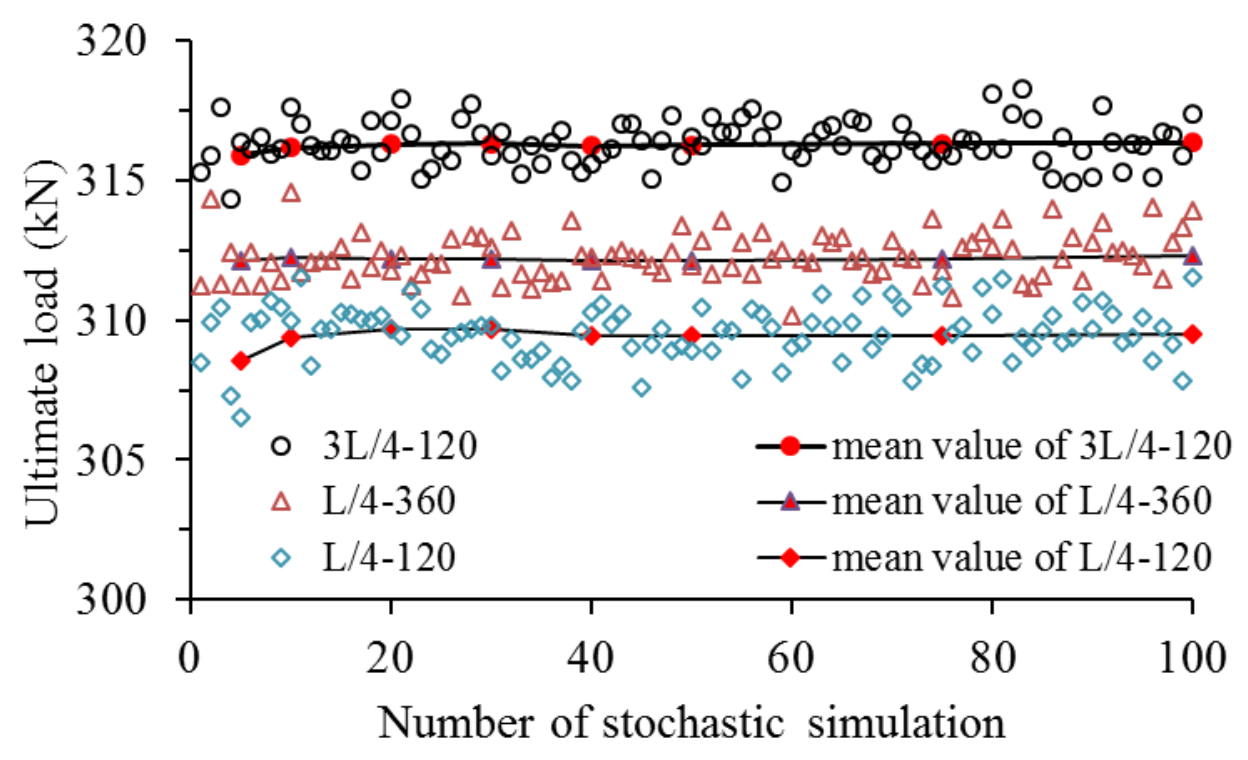

Figure 7 Convergence of stochastic simulation on the tested pitted members. 
(a)
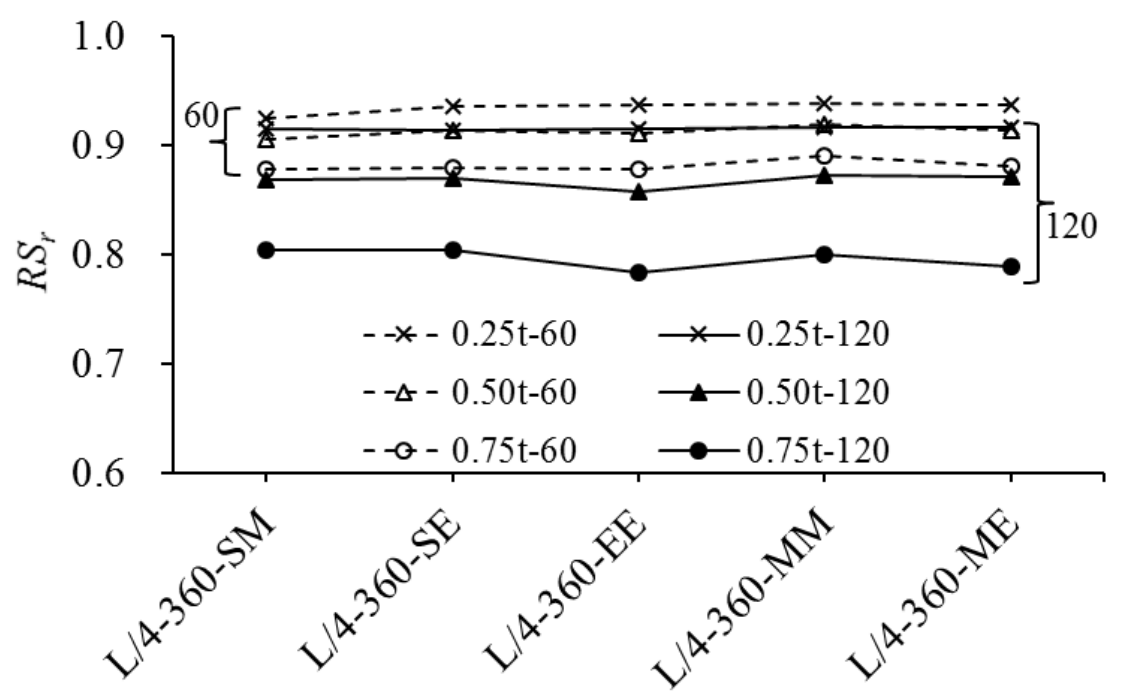

(b)

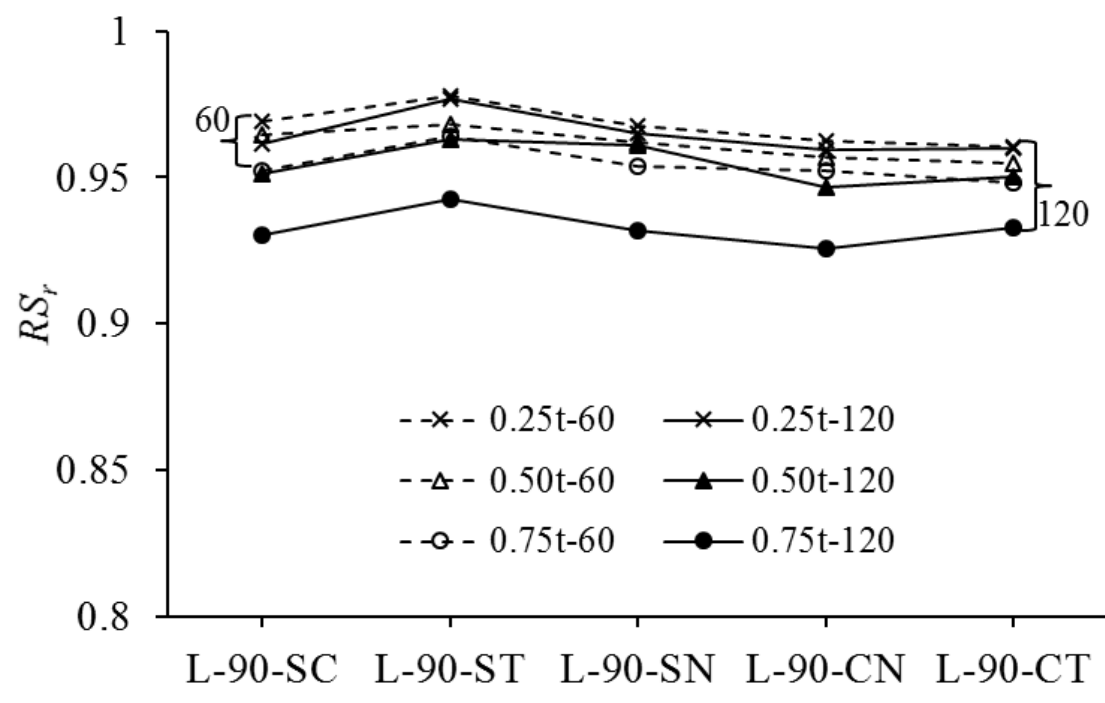

Figure 8 Variation of ultimate strength due to location change of corrosion patches in short columns.

(a) Change along the length; (b) change along the hoop.

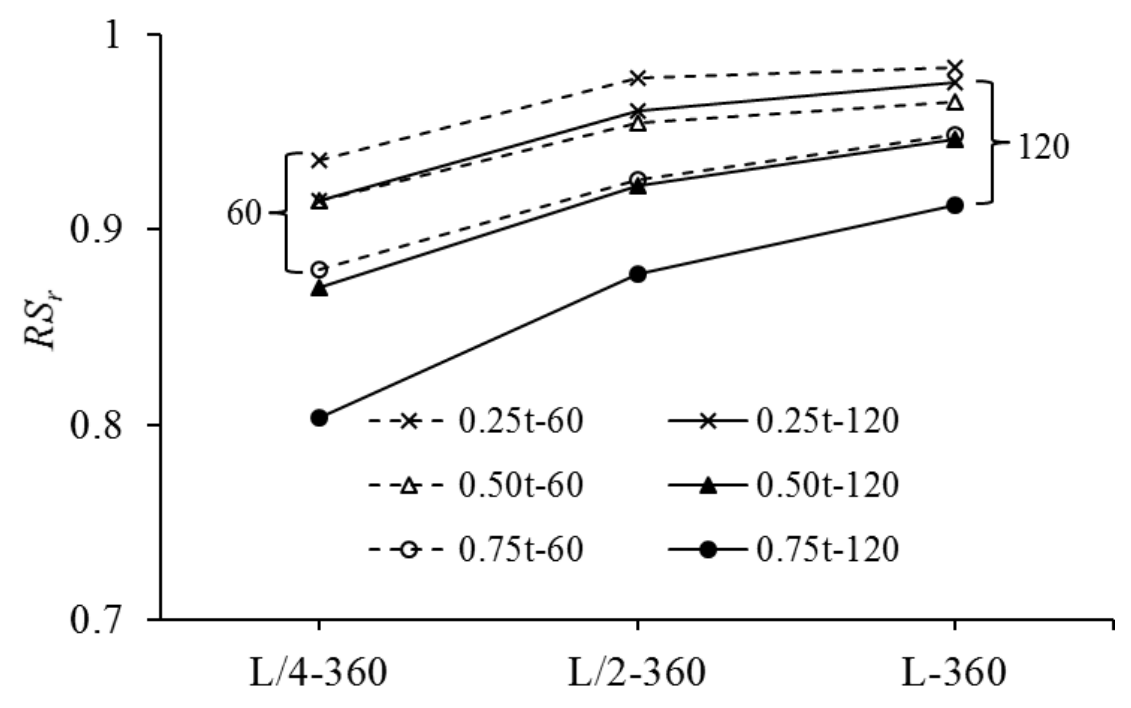




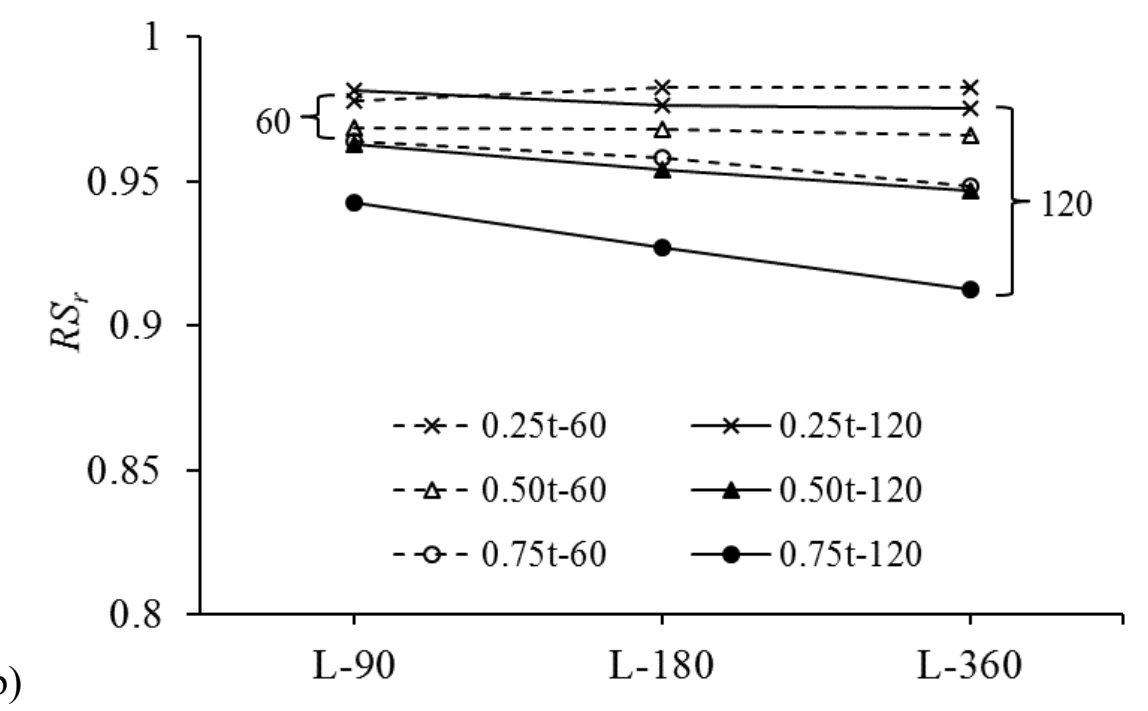

Figure 9 Variation of ultimate strength due to size change of corrosion patches in short columns. (a) Length change; (b) width change.

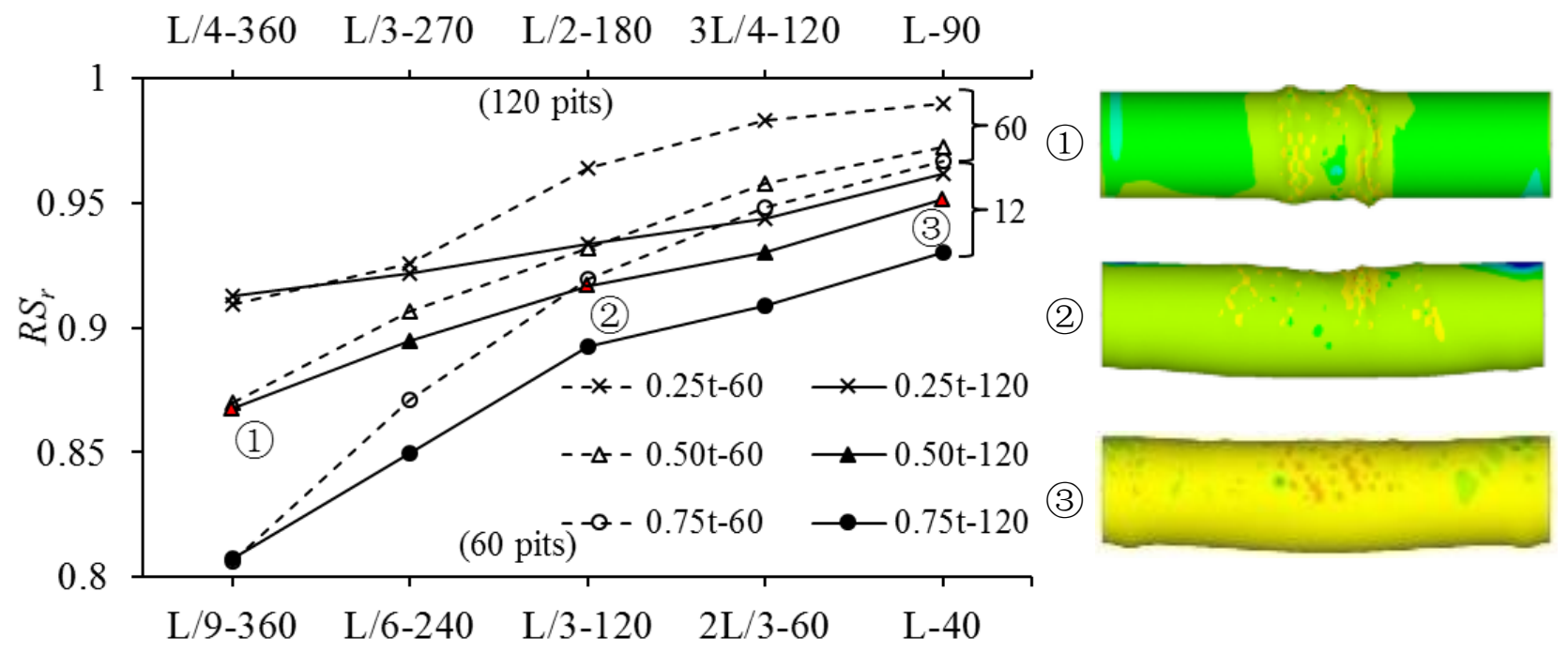

Figure 10 Variation of ultimate strength due to shape change of corrosion patches in short columns.

(a)

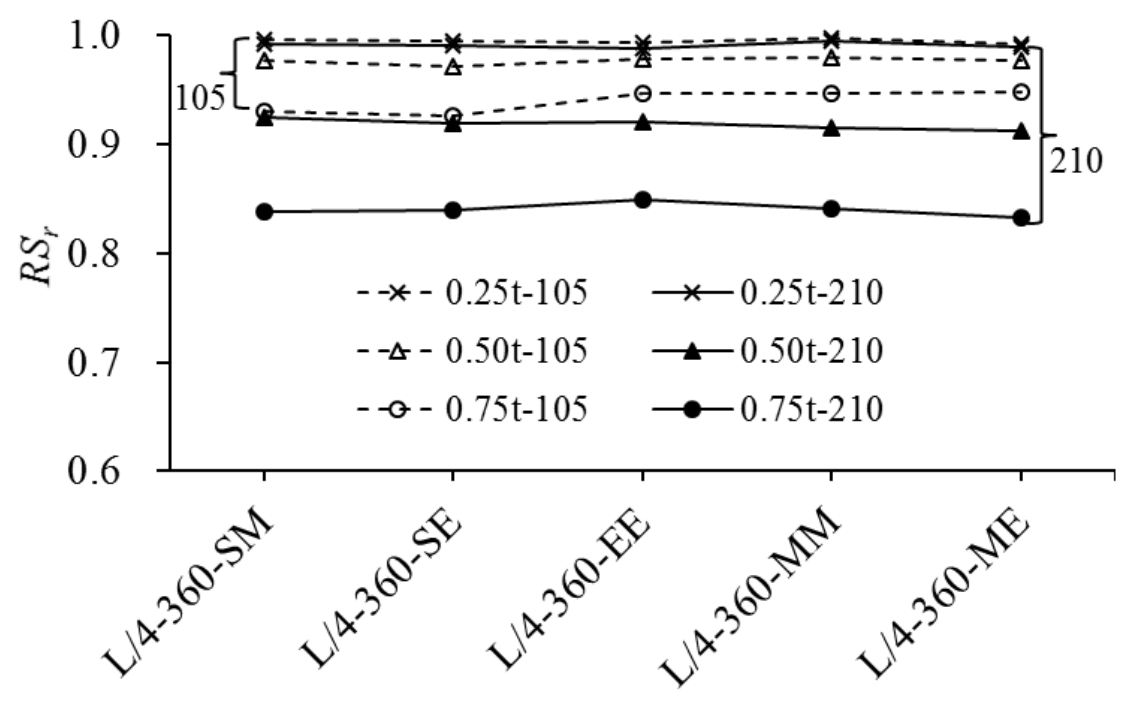




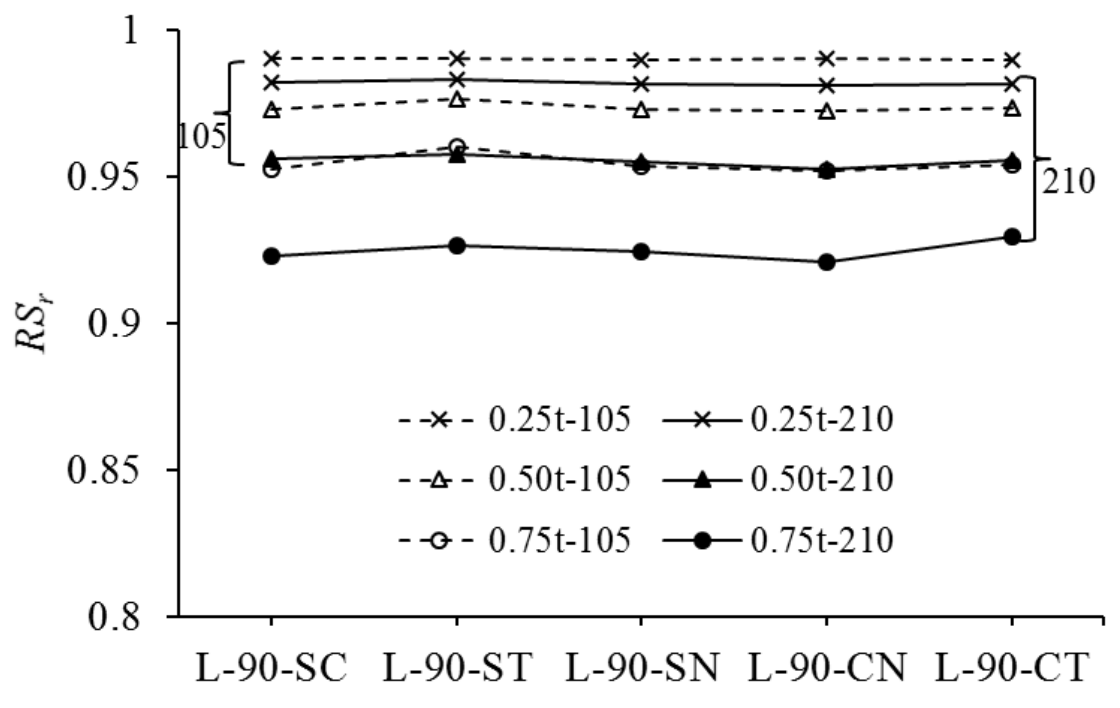

Figure 11 Variation of ultimate strength due to location change of corrosion patches in medium length columns. (a) Change along the length; (b) change along the hoop.

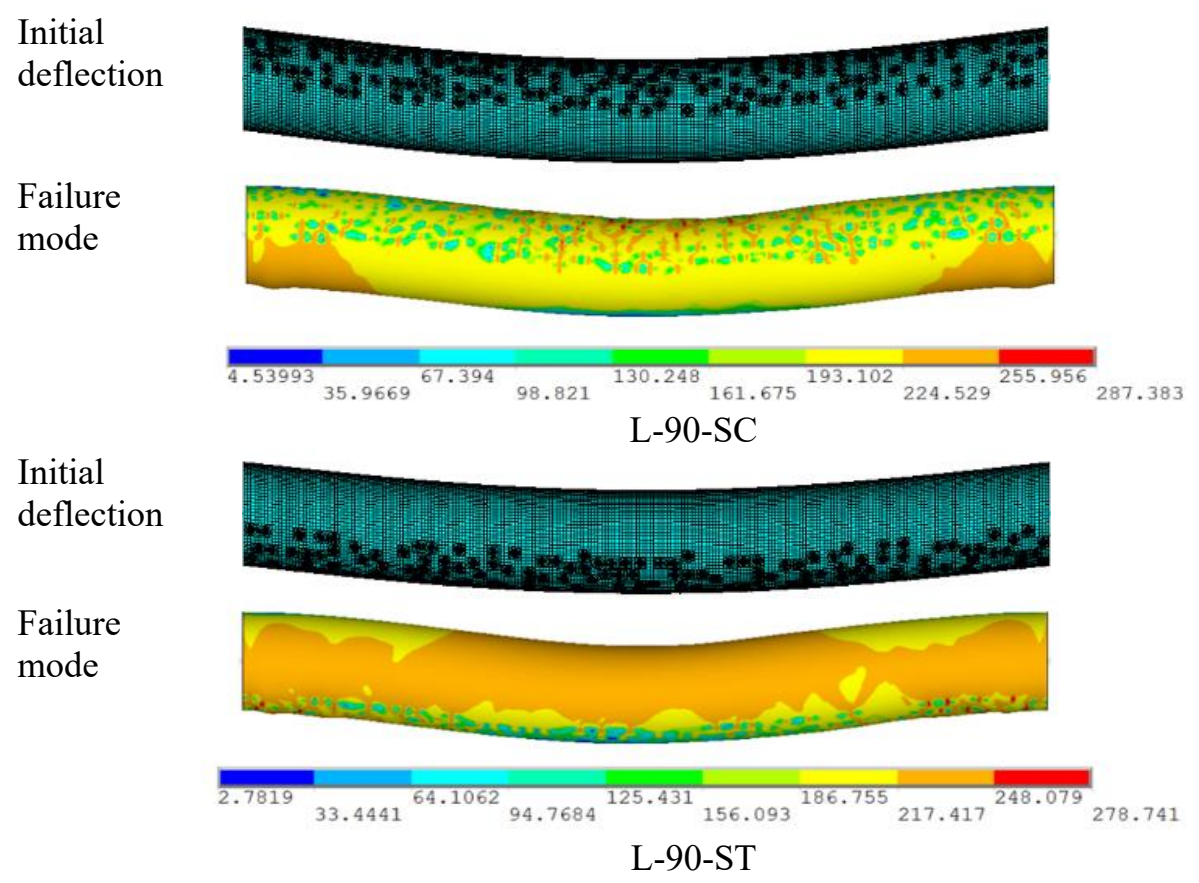

Figure 12 Failure mode dominated by initial deflection of a medium length column. 


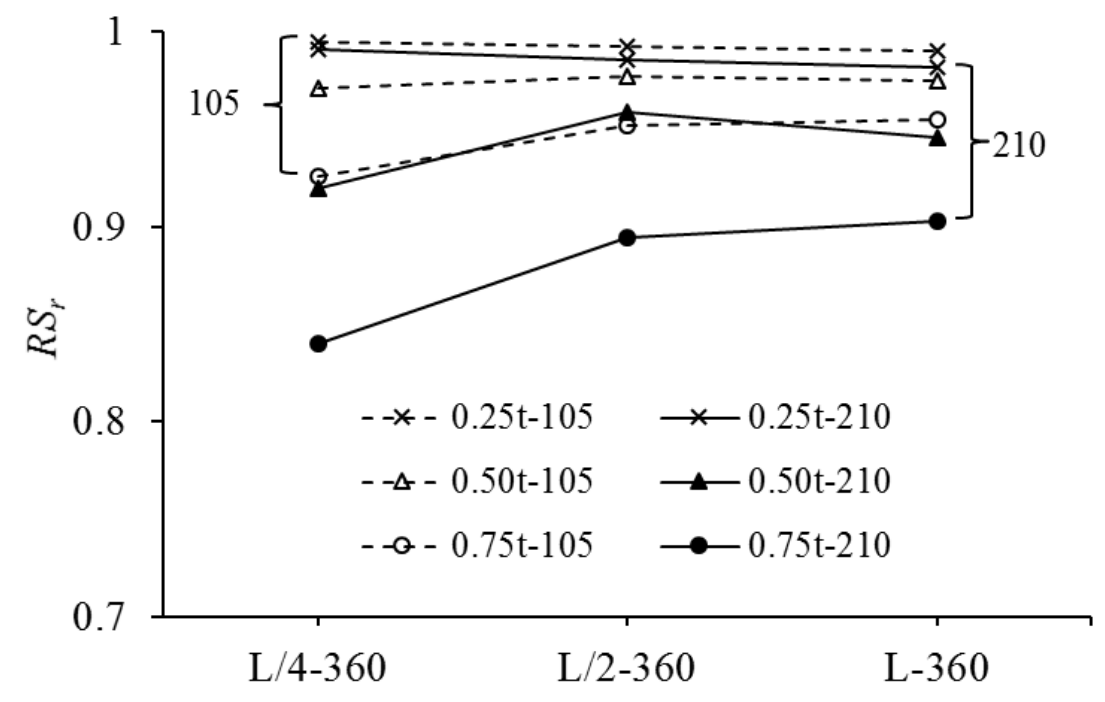

(a)

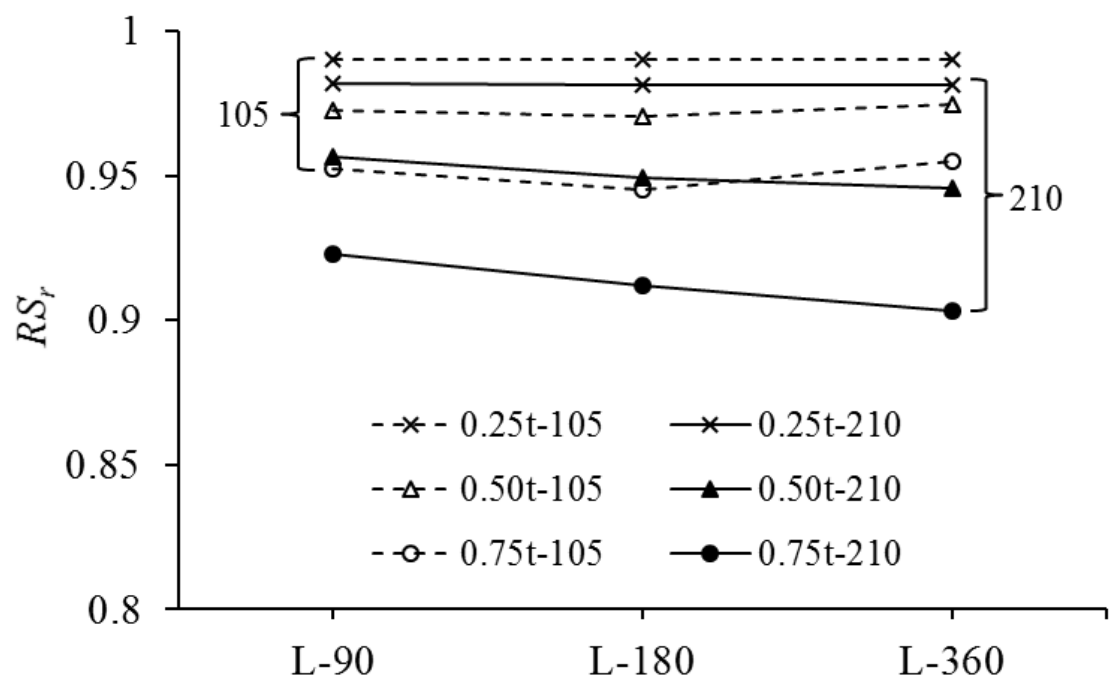

(b)

Figure 13 Variation of ultimate strength due to size change of corrosion patches in medium length columns. (a) Length change; (b) width change.

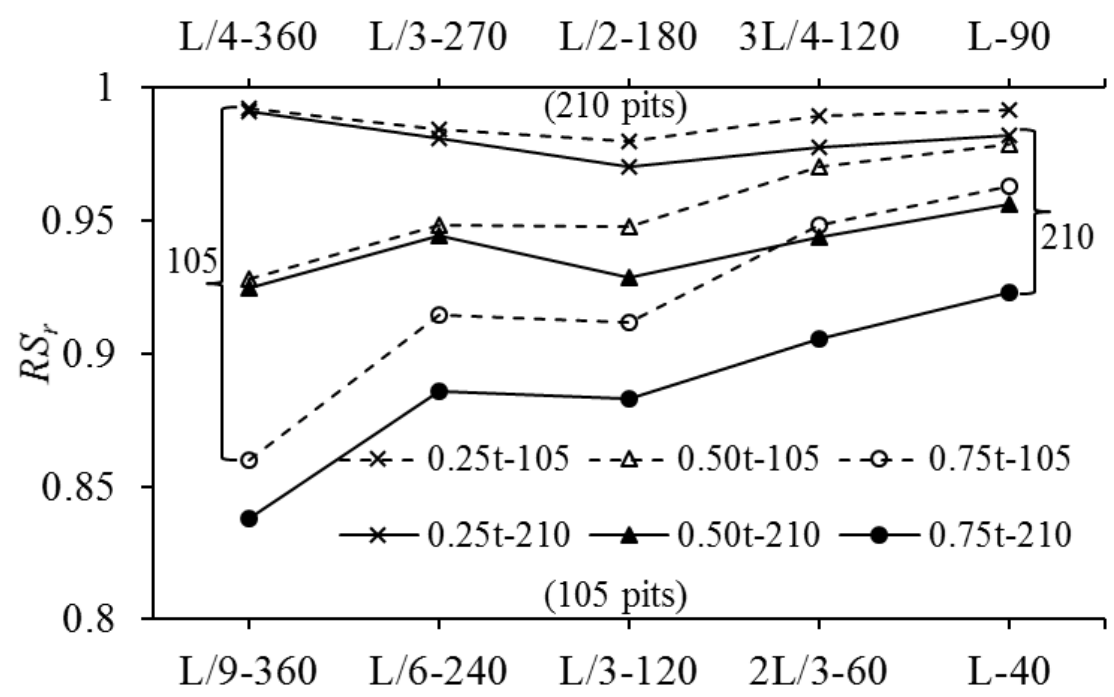

Figure 14 Variation of ultimate strength due to shape change of corrosion patches in medium length 
columns.

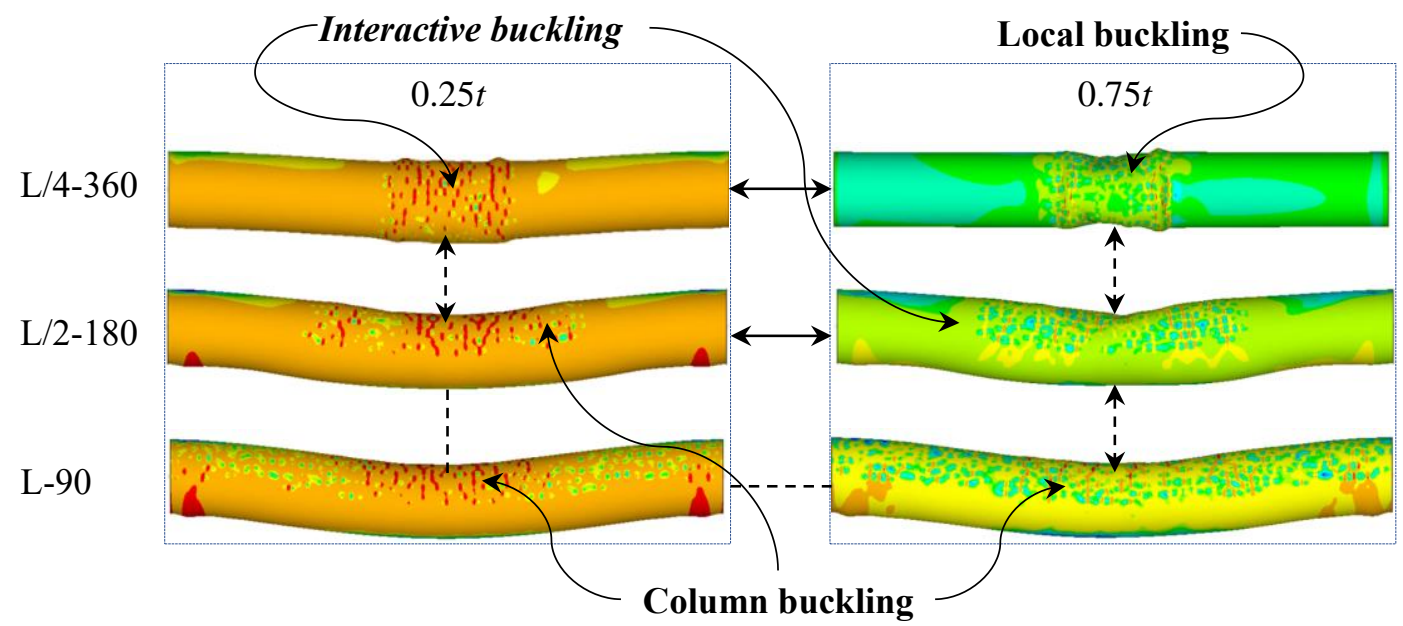

Figure 15 Failure mode transition due to shape change of corrosion patches in a medium length column. 

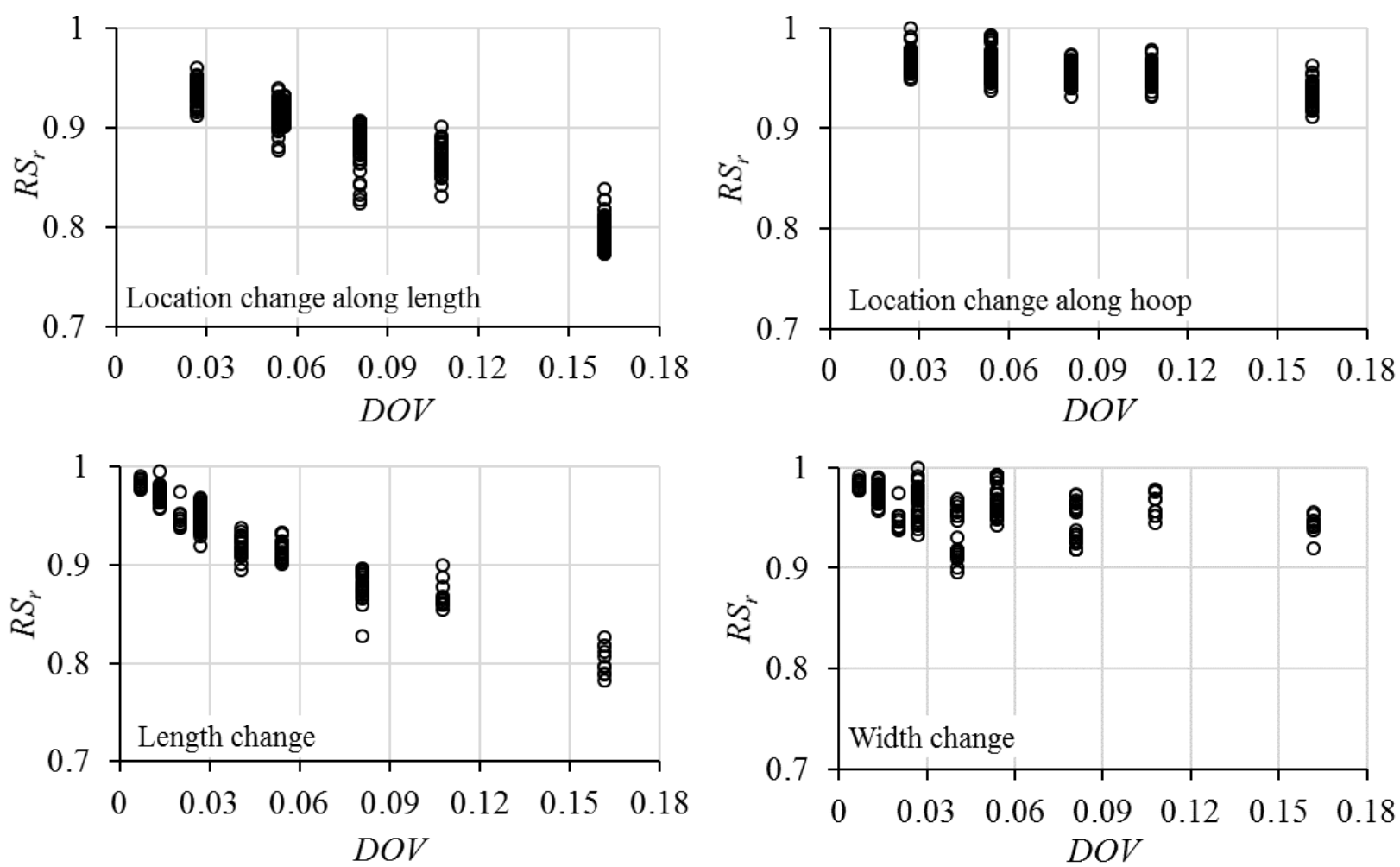

Figure 16 Variation of ultimate strength due to changes of location and size of corrosion patches in short columns.
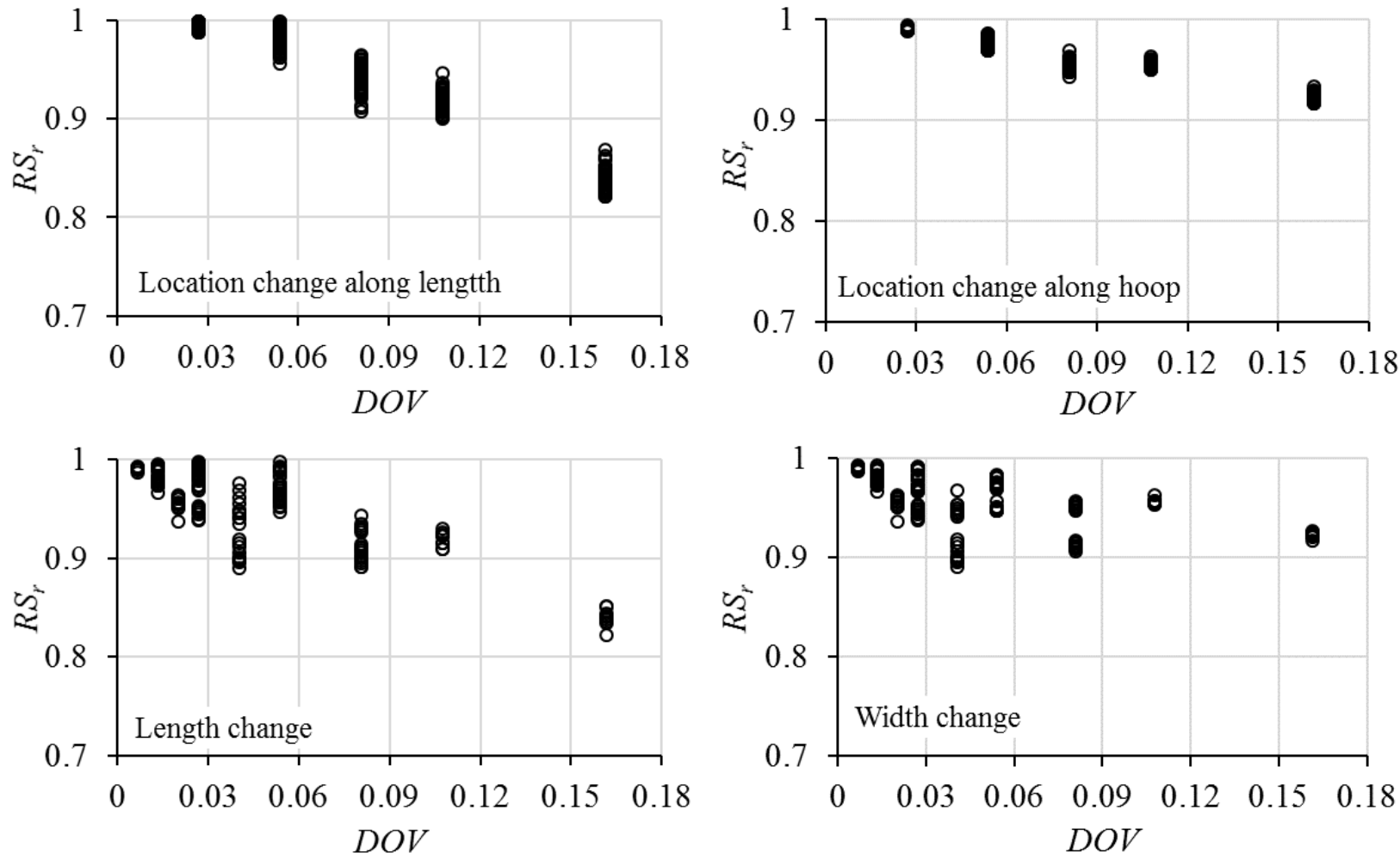

Figure 17 Variation of ultimate strength due to changes of location and size of corrosion patches in medium length columns. 

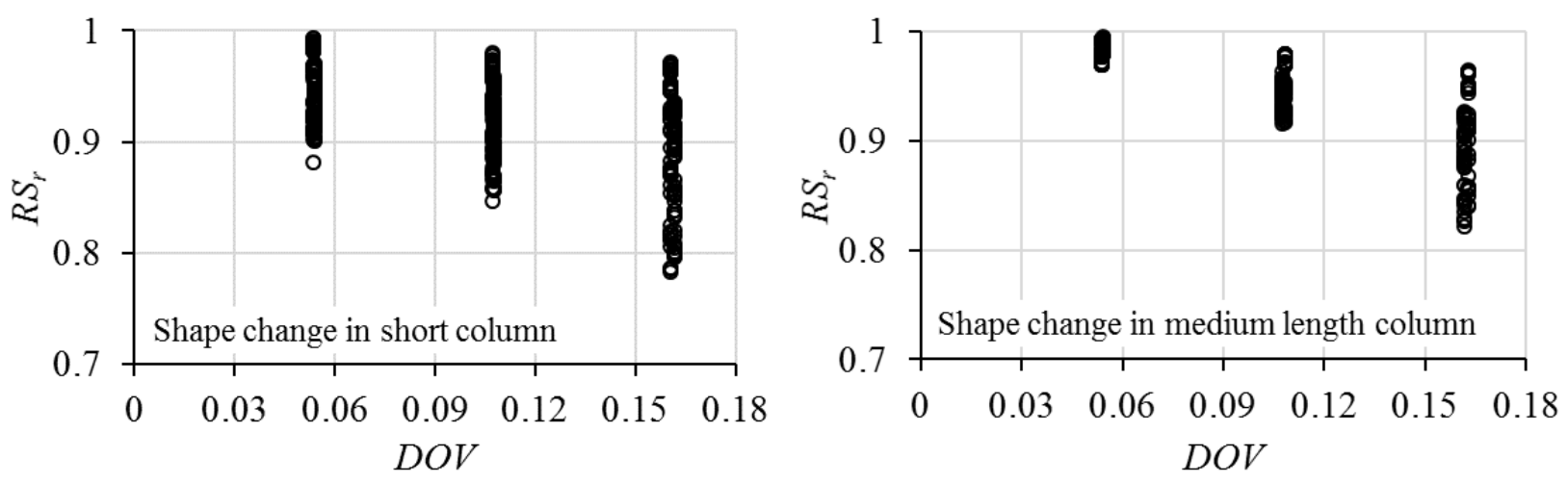

Figure 18 Variation of ultimate strength due to shape change of corrosion patches.

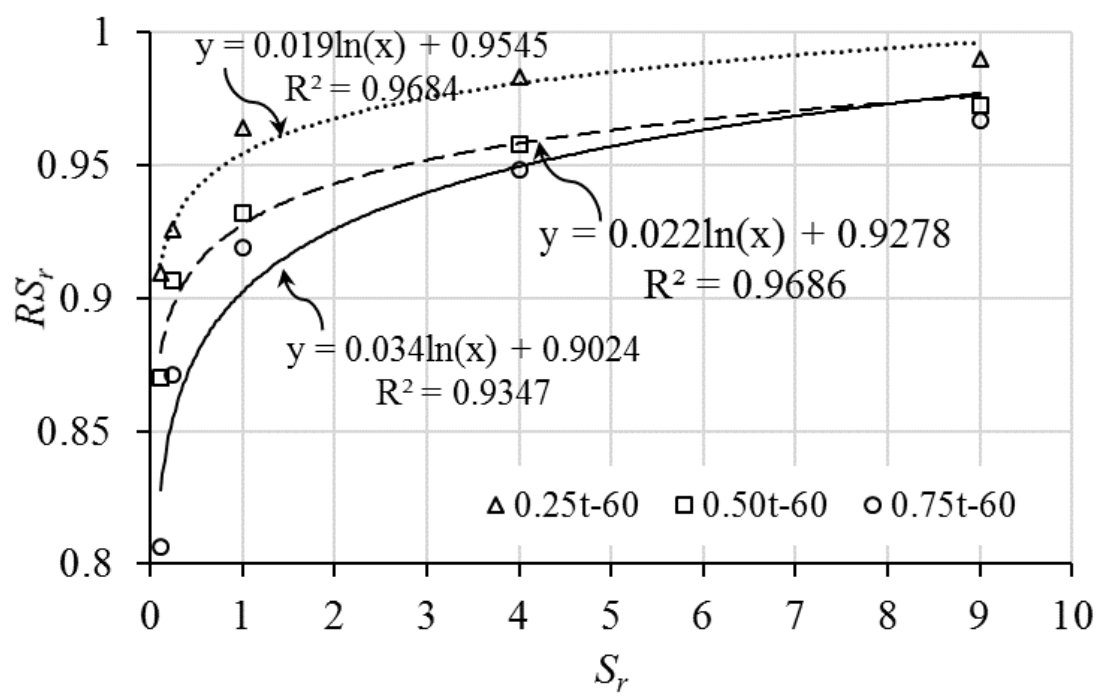

(a)

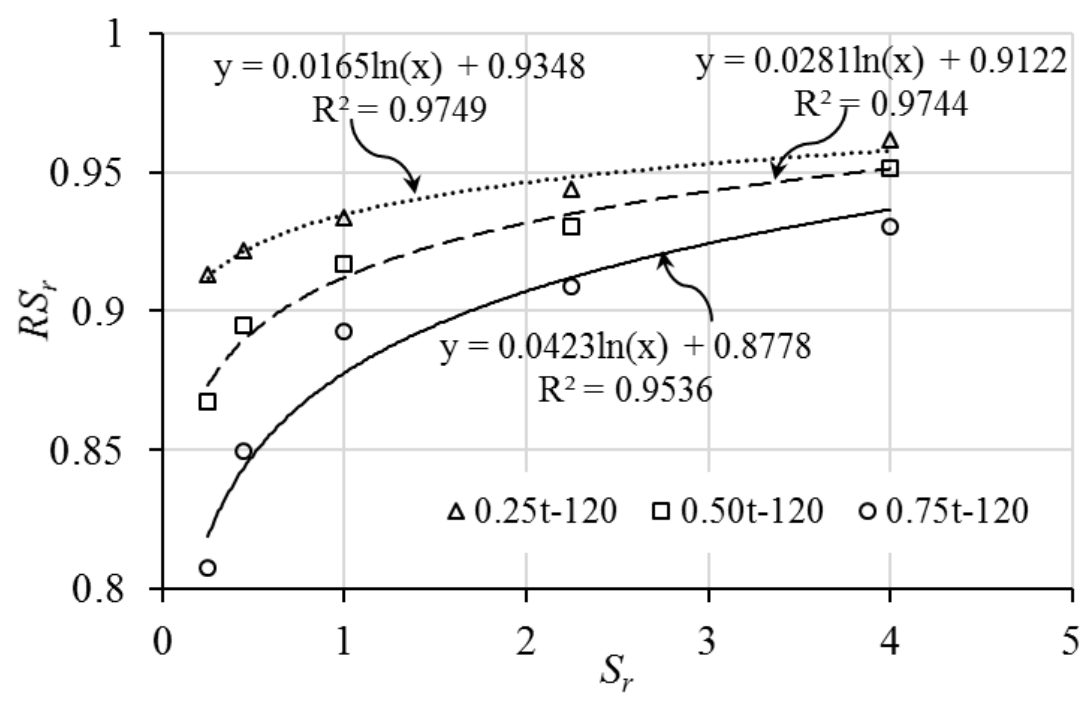

Figure 19 Relation of ultimate strength of short columns with shape ratios of corrosion patches. (a) $\pi D L / 9$ (60 pits); (b) $\pi D L / 4$ (120 pits). 


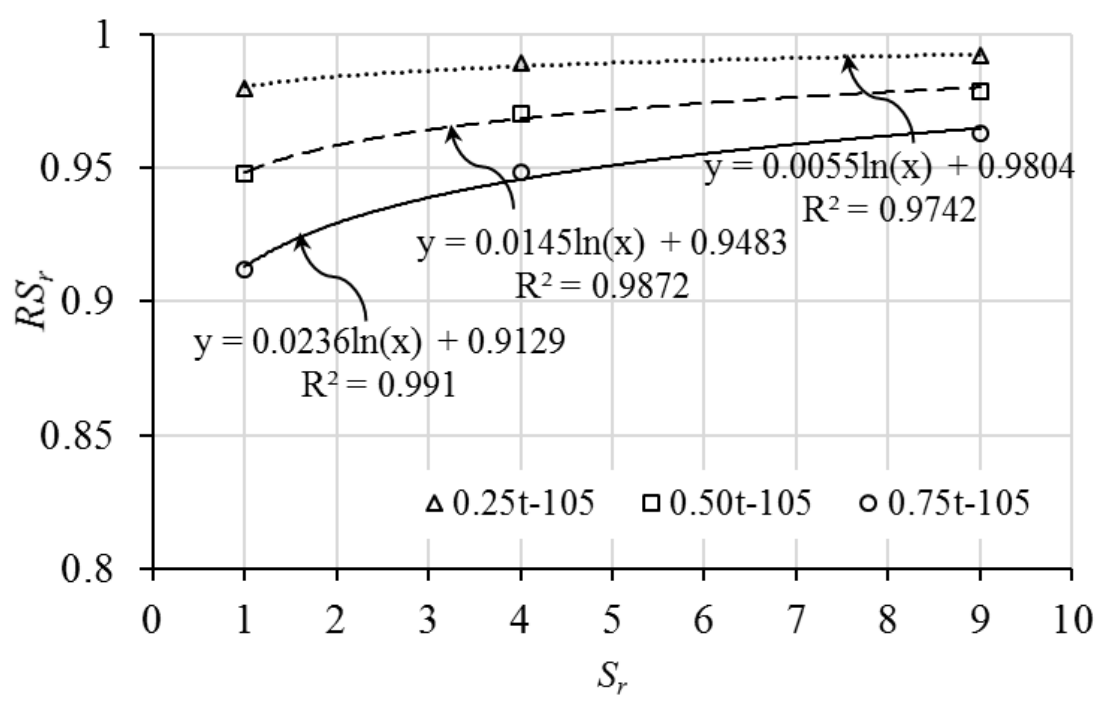

(c)

(d)

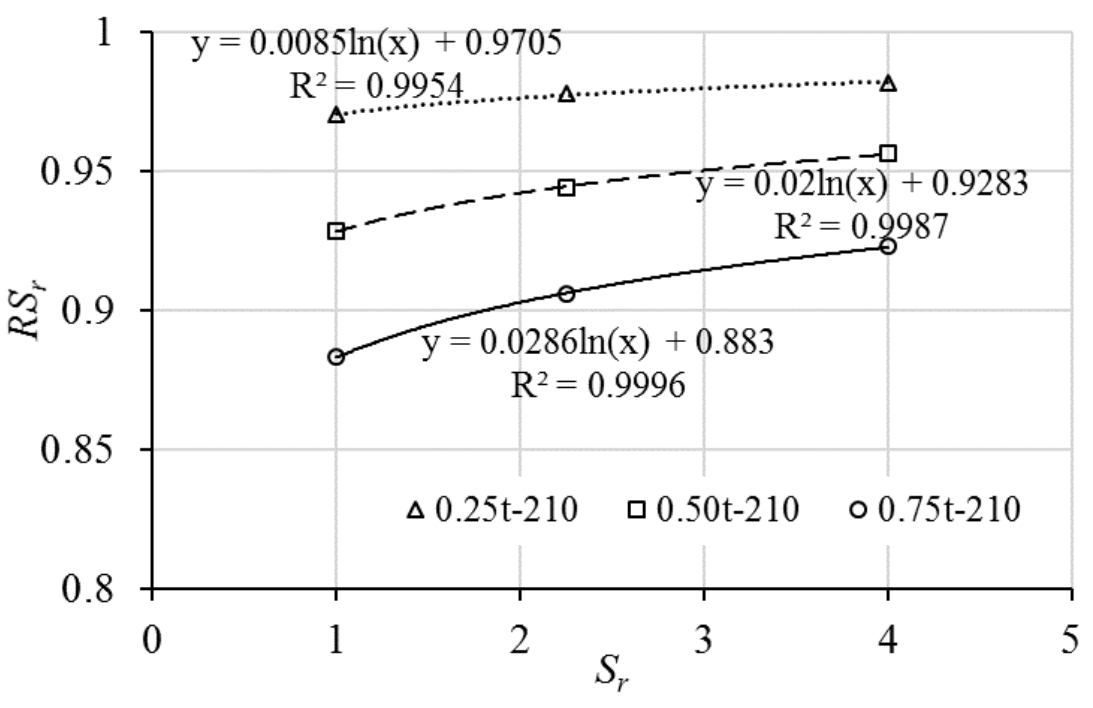

Figure 20 Relation of ultimate strength of medium length columns with shape ratios of corrosion patches. (a) $\pi D L / 9$ (105 pits); (b) $\pi D L / 4$ (210 pits).

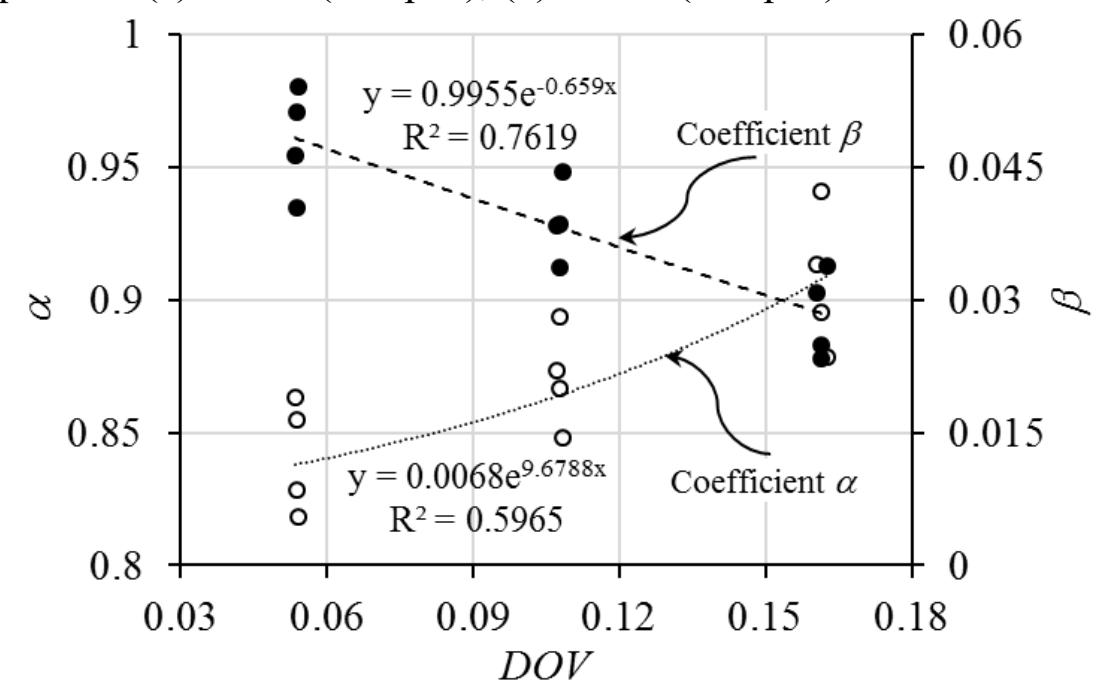

Figure 21 Coefficients of empirical formula of ultimate strength. 


\section{Tables}

Table 1 Test specimens with/without corrosion pits.

\begin{tabular}{cccccccccccc}
\hline Test & Specimen & $\begin{array}{c}L \\
(\mathrm{~mm})\end{array}$ & $\begin{array}{c}D \\
(\mathrm{~mm})\end{array}$ & $\begin{array}{c}t \\
(\mathrm{~mm})\end{array}$ & $L / D$ & $\lambda$ & $K$ & $\begin{array}{c}r \\
(\mathrm{~mm})\end{array}$ & $\begin{array}{c}d \\
(\mathrm{~mm})\end{array}$ & $\begin{array}{c}\text { DOP } \\
(\%)\end{array}$ & $\begin{array}{c}\text { DOV } \\
(\%)\end{array}$ \\
\hline 1 & $0-0$ & 480 & 59.8 & 4.33 & 8.0 & 12.2 & 0 & & & & \\
2 & $\mathrm{~L} / 4-120$ & 480 & 60.0 & 4.42 & 8.0 & 12.2 & 40 & 3 & 2.2 & 15.0 & 7.5 \\
3 & $3 \mathrm{~L} / 4-120$ & 480 & 60.0 & 4.39 & 8.0 & 12.2 & 120 & 3 & 2.1 & 15.0 & 7.3 \\
4 & $\mathrm{~L} / 4-360$ & 480 & 59.9 & 4.33 & 8.0 & 12.2 & 120 & 3 & 1.9 & 15.0 & 6.5 \\
\hline
\end{tabular}

Table 2 Results of stochastic simulation on ultimate strength $(\mathrm{kN})$ of test specimens.

\begin{tabular}{cccccccccc}
\hline \multirow{2}{*}{ Statistics } & Specimen & \multicolumn{7}{c}{ Interval runs } \\
\cline { 3 - 10 } & & 5 & 10 & 20 & 30 & 40 & 50 & 75 & 100 \\
\hline \multirow{4}{*}{$\mu$} & $1 / 4-120$ & 309 & 309 & 310 & 310 & 309 & 309 & 309 & 310 \\
& $3 / 4-120$ & 316 & 316 & 316 & 316 & 316 & 316 & 316 & 316 \\
& $1 / 4-360$ & 312 & 312 & 312 & 312 & 312 & 312 & 312 & 312 \\
\hline \multirow{4}{*}{$\sigma$} & $1 / 4-120$ & 1.67 & 1.44 & 1.17 & 1.01 & 1.03 & 0.99 & 0.99 & 0.97 \\
& $3 / 4-120$ & 1.21 & 0.97 & 0.79 & 0.84 & 0.79 & 0.76 & 0.73 & 0.77 \\
& $1 / 4-360$ & 1.33 & 1.27 & 0.94 & 0.86 & 0.85 & 0.79 & 0.78 & 0.83 \\
\hline
\end{tabular}

Table 3 Model parameters of tubular sections in numerical analyses.

\begin{tabular}{ccccccccc}
\hline Member & $L(\mathrm{~mm})$ & $D(\mathrm{~mm})$ & $t(\mathrm{~mm})$ & $L / D$ & $\lambda$ & $K$ & $r(\mathrm{~mm})$ & $d(\mathrm{~mm})$ \\
\hline short & 1200 & 267.4 & 9.3 & 4.5 & 6.6 & 60,120 & 12 & $0.25 t, 0.5 t, 0.75 t$ \\
medium & 2100 & 267.4 & 9.3 & 7.9 & 11.5 & 105,210 & 12 & $0.25 t, 0.5 t, 0.75 t$ \\
Material properties: & & & & & & \\
$E=206 \mathrm{GPa} ; v=0.3 ; \sigma_{s}=216 \mathrm{MPa} ; \sigma_{u}=373 \mathrm{MPa} ; \varepsilon_{u}=0.2$.
\end{tabular}

Table 4 Comparison between predicted and experimental results.

\begin{tabular}{cccccc}
\hline Specimen & DOV $(\%)$ & $S_{r}$ & Tested $R S_{r}$ & Analytical $R S_{r}$ & Relative error (\%) \\
\hline $1 / 4-120$ & 8.14 & 0.75 & 0.925 & 0.939 & 1.53 \\
$1 / 4-360$ & 6.95 & 0.25 & 0.961 & 0.932 & -2.99 \\
3/4-120 & 7.86 & 2.25 & 0.921 & 0.957 & 3.90 \\
D-FPT3 [18] & 7.5 & 0.25 & 0.898 & 0.928 & 3.34 \\
FP-CH300T3 [18] & 7.5 & 0.25 & 0.908 & 0.928 & 2.20 \\
HP-CH300T3 [18] & 3.7 & 0.5 & 0.899 & 0.965 & 7.32 \\
\hline
\end{tabular}

